\title{
Static and dynamic multipolar field expansions of dislocations and cracks in solids
}

\author{
B. Gurrutxaga-Lerma \\ Trinity College, University of Cambridge CB2 1TQ Cambridge, UK and \\ Department of Engineering, University of Cambridge, Cambridge, UK*
}

\begin{abstract}
This article provides a comprehensive analysis of the way multipolar field expansions, of common use in the study of point defects in solids, may be extended to study the long range fields of dislocations and cracks. The long range fields of such defects are of relevance in fields as disparate as dislocation dynamics, microcrack and fragmentation, or radiation damage studies. The article provides a general framework for the development of multipolar field expansions in the continuum; one that may be used for any generalised force distribution. The general framework is combined with the Burridge-Knopoff force representation of dislocations and cracks, both in the planar and in the three dimensional cases, to achieve their respective multipolar field expansions for generalised dislocation loop and crack geometries. It is shown that, despite its simplicity, the multipolar field expansions provide a very accurate measure of the far field of both planar and three dimensional dislocations and cracks, and that the accuracy increases as higher order terms (i.e., quadrupolar, octopolar, etc) are introduced into the expansion. The formulation is then extended to the elastodynamic case. Both a spatial-temporal multipolar field expansion and a spatial multipolar field expansion, are developed. The spatial-temporal multipolar field expansion is seen to capture only the leading terms of the elastodynamic fields, whilst the spatial multipolar expansions are seen to be very accurate at capturing the long range field behaviour so long as the characteristic speed of the dislocation or crack are a fraction of the longitudinal speed of sound.
\end{abstract}

\section{INTRODUCTION}

This article concerns the multipolar field expansions of the elastic fields of dislocations and cracks. By the multipolar expansion of an elastic field we mean the approximation of that same elastic field as a set of force multipoles which are applied over a single point, their distributional complexity increasing with the expansion's order, and their magnitude dependent on the actual field's underlying characteristics. Thus, an otherwise arbitrary and extended elastic field, as would be that of a dislocation loop or a three dimensional crack, may be approximated as a set of point force dipoles, quadrupoles, octopoles, etc, applied on a single point. The result of the expansion is meant to be an approximation that captures correctly the energetics and the long range behaviour of the elastic field, even if the near field is incorrectly approximated. Because it is the result of the application of simple point forces of constant albeit welldefined magnitude, the multipolar expansion will generally be simpler to evaluate than the original elastic field.

Multipolar expansions of this sort were originally developed in electrodynamics, for the study of long range electromagnetic fields of charge distributions [1]: by approximating the long range potential as if they originated on a multipole of point charges, one can greatly simplify the study of the distribution's long range effects. This has proven to be a fruitful avenue of research, particularly in the study of the electronic structure of complex molecules [2], and in spectroscopy [3], amongst many other examples.

One of the classical results derived from the study of crystal defects in elastic solids is that of the force multipole models of point defects. As in the electrodynamic case, this is aimed at producing an elastic model of the point defect as a set of forces (see for instance $[4,5]$ ), and at being able to study their long range effects and interactions from an energetically consistent perspective. This article generalises this approach to the modelling of dislocations and cracks. This generalisation is based on one of the crucial results by Backus and Mulcahy [6, 7], namely that the moment tensor and the force representations of an elastic source can be given in terms of their 'polynomial moments'. In parallel, and within the context of non-linear elasticity, Kunin [8] showed that the fields of arbitrary inhomogeneities may be expressed in terms of perturbations of the boundary conditions, that enabled expressing the fields of the defect as a series expansion reliant on the homogeneous, infinite medium's Green's function.

Traditionally, the use of multipolar expansions in the theory of dislocations was associated with the use of clusters of dislocations (e.g., dipoles, quadrupoles,... of edge or screw dislocations) as a way of computing their collective long range effects on other such clusters [9-11]. In addition, based on Kunin's

\footnotetext{
*bg374@cam.ac.uk
} 
theory, Kosheleva and coworkers $[12,13]$ applied multipolar field expansions to the theory of composite media.

In recent years, a number of more sophisticated multipolar expansions related to the computation of dislocation interactions $[14,15]$ and the elastic fields of dislocations $[16,17]$ have been offered in the context of extending the fast multipole technique [18] to dislocation dynamics simulations and dislocation theory; in this way, they offer a considerable speed up factor compared to conventional discrete dislocation dynamics methods, and enable more cost effective theoretical treatment of the long range interactions between dislocations and other crystalline defects[17]. As opposed to the full multipolar expansion offered in this article, these methods are based on the dipolar field expansion of the elastic energy[14, 15] and the stress tensor [16]; they are, therefore, multipolar expansions of the moment tensors, based on De Wit's line integral formalism [19]. Furthermore, other formulations of multipolar field expansions have recently been employed to study the far field asymptotic field of the elastic fields generated by inhomogeneities[20], and in the context of homogenisation theory[21].

This article generalises these efforts, and extends them to higher order multipolar moments, to elastic anisotropy, and to elastodynamics, offering a comprehensive theoretical framework with which to develop further multipolar expansions of extended defects, as we do for cracks in this work. In both the static and elastodynamic cases, we show that the multipolar field expansions correctly capture the long range behaviour of dislocations and cracks, to increasing accuracy as the multipolar moments' order is increased. As will be seen, by applying the representation theorem we make the multipolar expansion generally anisotropic, and dependent on Green's tensor function and the dislocation's or crack's moments of area. We argue that such expansions are of relevance to the study of either defect when their representative lengths are small compared with the scale of the problems. For dislocations, this could refer to the study of nano-loops resulting from radiation damage, or to the study of the collective behaviour of dislocations in an statistical manner. For cracks, the formulation we present would be of relevance to all those applications where microcracks are present, particularly if their numbers are large: from damage in semiconductors, to fragmentation studies, in all those cases small cracks will interact with one another not so much via their near fields but through their long range fields, and the multipolar field expansions we introduce in this article offer an exact and cost-effective description of these very long range effects.

The methodology introduced in this article relies on employing the dislocation's and crack's force representation, as opposed to their eigenstrain or eigenstress representations. The need for a force representation arises in section II, where we offer the multipolar field expansion of a general displacement field. We shall see that, in the same way the multipolar field expansions of point defects require their description as sets of forces, the true multipolar field expansion of any other extended defect requires it too. Thus, in section III we provide the elastostatic force representation of both dislocations and cracks by means of the Burridge-Knopoff theorem[22], which we briefly discuss. This formulation will provide the way of attaining higher order multipolar moments. Having defined the general expression of a multipolar field expansion and provided the force representation of a general dislocation loop, in section IV we develop the theory of multipolar expansions of loop. We show that in the most general case of a dislocation loop, the multipolar field expression depends to increasing orders on the moments of area enclosed by the loop, leading to a simple and cost-effective formulation of the long range effects of dislocations. In section VI, we apply the very same formulation to cracks in a number of modes and geometries; albeit the number of cases considered here is non-exhaustive, we show the procedure to develop the multipolar field expansion of any one crack. In section VII we go on to extend the formulation to the case of elastodynamic, multipolar field expansions both for dislocations and cracks.

\section{MULTIPOLAR EXPANSION OF A FORCE DISTRIBUTION}

As a first step towards offering a complete description of the multipolar field expansions of defects, in this section we define the general formulation of the multipolar field expansion. Rather than follow Backus and Mulcahy[6] in defining the multipolar moments a priori, here we begin by considering a general body force distribution $f_{p}(\boldsymbol{x})$ acting as a source in a linear elastic, anisotropic, and unbounded solid. The displacement $u_{i}(\boldsymbol{x})$ field associated with the force distribution may be obtained as follows via the representation theorem [23]

$$
u_{i}(\boldsymbol{x})=\int_{\Omega^{\prime}} G_{i p}\left(\boldsymbol{x}-\boldsymbol{x}^{\prime}\right) f_{p}\left(\boldsymbol{x}^{\prime}\right) \mathrm{d} \Omega^{\prime}
$$

where $G_{i p}\left(\boldsymbol{x}-\boldsymbol{x}^{\prime}\right)$ is the elastic Green's function of an unbounded solid, and where repeated index denotes summation. 
We expand $G_{i p}\left(\boldsymbol{x}-\boldsymbol{x}^{\prime}\right)$ in Taylor series of $\boldsymbol{x}^{\prime}$ about 0 , rendering

$$
\begin{aligned}
G_{i p}\left(\boldsymbol{x}-\boldsymbol{x}^{\prime}\right) & =G_{i p}(\boldsymbol{x})-\frac{\partial G_{i p}(\boldsymbol{x})}{\partial x_{k_{1}}} x_{k_{1}}^{\prime}+\frac{1}{2 !} \frac{\partial^{2} G_{i p}(\boldsymbol{x})}{\partial x_{k_{1}} \partial x_{k_{2}}} x_{k_{1}}^{\prime} x_{k_{2}}^{\prime}-\frac{1}{3 !} \frac{\partial^{3} G_{i p}(\boldsymbol{x})}{\partial x_{k_{1}} \partial x_{k_{2}} \partial x_{k_{3}}} x_{k_{1}}^{\prime} x_{k_{2}}^{\prime} x_{k_{3}}^{\prime}+\ldots \\
& =\sum_{n=0}^{\infty} \frac{(-1)^{n}}{n !} \frac{\partial^{n} G_{i p}(\boldsymbol{x})}{\partial x_{k_{1}} \ldots \partial x_{k_{n}}} x_{k_{1}}^{\prime} \cdot \ldots \cdot x_{k_{n}}^{\prime}
\end{aligned}
$$

Substituting this above, we find

$u_{i}(\boldsymbol{x})=\int_{\Omega^{\prime}} \sum_{n=0}^{\infty} \frac{(-1)^{n}}{n !} \frac{\partial^{n} G_{i p}(\boldsymbol{x})}{\partial x_{k_{1}} \ldots \partial x_{k_{n}}} x_{k_{1}}^{\prime} \cdot \ldots \cdot x_{k_{n}}^{\prime} f_{p}\left(\boldsymbol{x}^{\prime}\right) \mathrm{d} \Omega=\sum_{n=0}^{\infty} \frac{1}{n !} \frac{\partial^{n} G_{i p}(\boldsymbol{x})}{\partial x_{k_{1}} \ldots \partial x_{k_{n}}} \int_{\Omega^{\prime}}\left[x_{k_{1}}^{\prime} \cdot \ldots \cdot x_{k_{n}}^{\prime} f_{p}\left(\boldsymbol{x}^{\prime}\right)\right] \mathrm{d} \Omega^{\prime}$

We then identify, in direct analogy to the point defect's case (see [24]), the force multipole moment associated with force distribution as

$$
\gamma_{p k_{1} \ldots k_{n}}^{(n)}=\int_{\Omega^{\prime}} x_{k_{1}}^{\prime} \cdot \ldots \cdot x_{k_{n}}^{\prime} \cdot f_{p}\left(\boldsymbol{x}^{\prime}\right) \mathrm{d} \Omega^{\prime}
$$

where $n$ denotes the order of the multipolar moment, so that the displacement field associated with the multipole of order $n$ is then given by

$$
u_{i}(\boldsymbol{x})=\sum_{n=0}^{\infty} \frac{(-1)^{n}}{n !} \frac{\partial^{n} G_{i p}(\boldsymbol{x})}{\partial x_{k_{1}} \ldots \partial x_{k_{n}}} \gamma_{p k_{1} \ldots k_{n}}
$$

We note here that a similar expansion argument can be provided for the moment tensor representation, and obtain the moment tensor density multipole expansion instead. In that case, the higher order moments would be given by

$$
\kappa_{i p k_{1} \ldots k_{n}}^{(n)}=\int_{\Omega^{\prime}} x_{k_{1}}^{\prime} \cdot \ldots \cdot x_{k_{n}}^{\prime} \cdot m_{i p}\left(\boldsymbol{x}^{\prime}\right) \mathrm{d} \Omega^{\prime}
$$

where $m_{i p}$ is the moment tensor density. However, as was noted by Backus and Mulcahy [6], knowledge of a moment tensor multipolar moments does not uniquely determine the elastic field in a multipolar expansion, since

$$
\gamma_{p k_{1} \ldots k_{n}}^{(n)}=\sum_{\Pi} \kappa_{k_{1}^{\prime} p k_{2}^{\prime} \ldots k_{n}^{\prime}}^{(n-1)}
$$

where $\Pi$ is each permutation in the order of $B=\left\{k_{1}^{\prime}, \ldots, k_{n}^{\prime}\right\}$ relative to $A=\left\{k_{1}, \ldots, k_{n}\right\}$ such that $A=B$. Therefore, the moment multipole expansion seems less suitable for representing dislocations and other extended defects if higher order terms (i.e., quadrupolar and beyond) are required.

We also note that in expressing the displacement field as in eqn.5, each multipolar term can be regarded as being the result of the convolution of the field's Green function with a point force distribution applied at the origin. For instance, the first order, dipolar, moment term would arise if a force doublet of magnitude $\gamma_{p k_{1}}^{(1)}$, i.e., $f_{p}(\boldsymbol{x})=\gamma_{p k_{1}}^{(1)} \partial_{k_{1}} \delta(\boldsymbol{x})$, were applied along each cartesian direction at the origin; the convolution of this term with the Green's function via the representation theorem in eqn.1 renders

$$
u_{i}^{(1)}=\int_{\Omega^{\prime}} G_{i p}\left(\boldsymbol{x}-\boldsymbol{x}^{\prime}\right) \gamma_{p k_{1}}^{(1)} \partial_{k_{1}} \delta\left(\boldsymbol{x}^{\prime}\right) \mathrm{d} \Omega^{\prime}=\frac{\partial G_{i p}}{\partial x_{k_{1}}} \gamma_{p k_{1}}^{(1)},
$$

which is the first order term in the expansion in eqn.5. Analogously, the second order quadrupolar moments would correspond to the application of a force quadrupole of magnitude $\gamma_{p k_{1} k_{2}}^{(2)}$ at the origin, and so on. Thus, the multipolar field expansion subsumes any one force distribution into a series of force dipoles, quadrupoles, etc., applied at the origin. The calculation of a multipolar field expansion is the computation of the multipolar moments that provide the magnitude of those force multipoles at the origin. As can be seen in eqn.4, these multipolar moments are heavily influenced by the geometry of the force distribution. This stands in direct analogy to electrodynamics, where multipolar representations were first developed [1]. 


\section{A. Properties of the force multipole moments}

The force multipoles fulfil a number of symmetries and other properties. Thus, the $0^{\text {th }}$ order moment is just the total force exerted by the dislocation over the medium, which one would expect to be

$$
\gamma_{p}^{(0)}=0
$$

as required by equilibrium. We note that it can be checked that this is true for dislocation loops and straight screw dislocations, but that for straight edge dislocations, as we have discussed before, there exists a net force acting in the direction normal to the slipped surface, whereby $\gamma_{p}^{(0)} \neq 0$.

The $1^{\text {st }}$ order moment is related to force moments (i.e., torques). In particular, if we compare

$$
\gamma_{p k_{1}}^{(1)}=\int_{\Omega^{\prime}} x_{k_{1}}^{\prime} \cdot f_{p}\left(\boldsymbol{x}^{\prime}\right) \mathrm{d} \Omega^{\prime}
$$

with the total torque, $M_{j}$, exerted over the medium

$$
M_{j}=\int_{\Omega^{\prime}} \epsilon_{j k_{1} p} x_{k_{1}}^{\prime} \cdot f_{p}\left(\boldsymbol{x}^{\prime}\right) \mathrm{d} \Omega^{\prime}
$$

we see that $\epsilon_{j k_{1} p} \gamma_{p k_{1}}^{(1)}$ is the $j^{\text {th }}$ component of the total torque. Equilibrium in force moments therefore requires that $\gamma_{p k_{1}}^{(1)}=0$. We note that equilibrium in torques requires the topological closure of the defect, meaning that as explored in the following sections, neither straight edge nor straight screw dislocations on their own are equilibrated in moments. In fact, in principle equilibrium would imply the strong symmetry that

$$
\gamma_{p k_{1}}^{(1)}=\gamma_{k_{1} p}^{(1)}
$$

which straight dislocations do not generally fulfil. It is therefore a property of the straight dislocations that their force moment tensors are not symmetric.

Further symmetries exist. Crucially, it is clear from eqn.4 that the ordering of the $k_{1}, \ldots, k_{n}$ indices is irrelevant. That is, if the sets $A=\left\{k_{1}, \ldots, k_{n}\right\}$ and $B=\left\{k_{1}^{\prime}, \ldots, k_{n}^{\prime}\right\}$ are by extension $A=B$, then

$$
\gamma_{p k_{1} \ldots k_{n}}^{(n)}=\gamma_{p k_{1}^{\prime} \ldots k_{n}^{\prime}}^{(n)}
$$

\section{FORCE REPRESENTATIONS OF DISLOCATIONS AND CRACKS}

In section II we have the multipolar field expansion of a force distribution. Generally however, extended defects such as dislocations and cracks are defined not in terms of force distributions, but as eigenstrains $[23,24]$ or eigenstresses [6]. The latter two are but two of at least four different ways of representing sources in an elastic continuum, the other two being force representations [22] and moment tensors [7]. Following the formal definitions of the field multipolar moments given in sec II, it is clear that multipolar expansions require the description of the defect as body forces. This can be achieved both for dislocations and cracks by adapting the Burridge-Knopoff force representation theorem [22].

\section{A. Burridge-Knopoff force representation theorem}

The Burridge-Knopoff force representation theorem (hereafter, BK theorem) states that any internal discontinuity in the displacement or traction fields of a linear elastic solid may be represented by an equivalent body force distribution applied on a perfect elastic continuum, such that the elastic fields of the discontinuity and its force equivalent are the same. It is important to stress that the BurridgeKnopoff forces are fictitious forces, not actual body forces, that are applied on the undisturbed, defect-free, continuum; as such, they can be shown to be the continuum equivalent of the Kanzaki forces in crystalline defects $[24,25]$. Here, we introduce the BK theorem without proof.

Let $\left[u_{i}\right]\left(\boldsymbol{x}^{\prime}, t\right)$ be a general displacement discontinuity. Here $\boldsymbol{u} \equiv u_{i}$ denotes the displacement field, which is generally dependent on time $t \in \mathbb{R}$ and on a spatial position vector $\boldsymbol{x}^{\prime} \in \mathbb{R}^{3}$. Let $S_{\boldsymbol{x}^{\prime}}$ be the internal surface defined by the support of $\left[u_{i}\right]\left(\boldsymbol{x}^{\prime}, t\right)$; let $\boldsymbol{\nu} \equiv \nu_{j}$ be the normal vector to $S_{\boldsymbol{x}^{\prime}}$. The BK 
theorem states that the elastic fields due to $\left[u_{i}\right]\left(\boldsymbol{x}^{\prime}, t\right)$ are equivalent to those due to some force distribution $f \equiv f_{p}$, given by ${ }^{1}$

$$
f_{p}(\boldsymbol{x}, t)=-\int_{S_{\boldsymbol{x}^{\prime}}}\left[u_{i}\right]\left(\boldsymbol{x}^{\prime}, t\right) \nu_{j} C_{i j p q} \frac{\partial \delta\left(\boldsymbol{x}-\boldsymbol{x}^{\prime}\right)}{\partial x_{q}} \mathrm{~d} S_{\boldsymbol{x}^{\prime}}
$$

where $C_{i j p q}$ is the elastic constant tensor, $\delta(\cdot)$ is Dirac's delta function. For brevity, hereafter

$$
\frac{\partial \delta\left(\boldsymbol{x}-\boldsymbol{x}^{\prime}\right)}{\partial x_{q}}=\delta_{q}\left(\boldsymbol{x}-\boldsymbol{x}^{\prime}\right)
$$

\section{B. BK forces of dislocation loops}

Let us consider a closed dislocation loop defined by some closed curve $C$. The Burgers vector, conserved as required by Frank's rule[26], is given by the vector $\boldsymbol{B} \equiv B_{i}$. Let $D \in \mathbb{R}^{3}$ be the set of all points enclosed by $C$. We define the characteristic function ${ }^{2} \chi_{D}(\boldsymbol{x})$ associated with the loop as (q.v.[27])

$$
\chi_{D}(\boldsymbol{x})= \begin{cases}0 & \boldsymbol{x} \notin D \\ 1 & \boldsymbol{x} \in D\end{cases}
$$

We note that $\iota_{D}(\boldsymbol{x})=-\boldsymbol{\nu} \cdot \nabla \chi_{D}(\boldsymbol{x})$ is the (compact) surface delta function, and that therefore $\iota_{D}^{\prime}(\boldsymbol{x})=$ $\nabla^{2} \chi_{D}(\boldsymbol{x})[28]$. The discontinuity dislocation loop may then be modelled as

$$
\left[u_{i}\right]\left(\boldsymbol{x}^{\prime}\right)=B_{i} \chi_{D}\left(\boldsymbol{x}^{\prime}\right)
$$

The associated plastic distortion, $\beta_{i j}=\left[u_{i}\right]_{, j}$, renders Kröner's formula [29]

$$
\beta_{i j}\left(\boldsymbol{x}^{\prime}, t\right)=B_{i} \partial_{j} \chi_{D}\left(\boldsymbol{x}^{\prime}\right)=-B_{i} \nu_{j} \iota(\boldsymbol{x})
$$

The corresponding force representation of the loop may then be found invoking the BK theorem as

$$
\begin{aligned}
f_{p}(\boldsymbol{x}) & =-\int_{D}\left[u_{i}\right](\boldsymbol{x}, t) \nu_{j} C_{i j p q} \delta_{q^{\prime}}\left(\boldsymbol{x}^{\prime}-\boldsymbol{x}\right) \mathrm{d} D=-C_{i j p q} B_{i} \frac{\partial}{\partial x_{q}} \int_{D} \nu_{j} \chi_{D}\left(\boldsymbol{x}^{\prime}\right) \delta\left(\boldsymbol{x}^{\prime}-\boldsymbol{x}\right) \mathrm{d} D \\
& =-C_{i j p q} B_{i} \nu_{j} \frac{\partial \chi_{D}(\boldsymbol{x})}{\partial x_{q}}
\end{aligned}
$$

This equation is the general force representation of an arbitrary dislocation loop described by the characteristic function $\chi_{D}(\boldsymbol{x})$, the support

a. Straight dislocations. Application of eqn.19 or, otherwise, directly of the BK theorem to the case where the loop degenerates in a straight edge or straight screw dislocation renders the force representation of straight dislocations. For the straight edge isotropic dislocation with Burgers vector along the $x_{1}$ axis, and dislocation line along the $x_{3}$ axis, using $C_{i j p q}=\lambda \delta_{i j} \delta_{p q}+\mu\left(\delta_{i p} \delta_{j q}+\delta_{i q} \delta_{j p}\right)$ eqn.19 renders

$$
f_{1}=-B \mu \partial_{x_{2}} \chi_{D}^{\text {edge }}(\boldsymbol{x}), \quad f_{2}=-B \mu \partial_{x_{1}} \chi_{D}^{\text {edge }}(\boldsymbol{x}), \quad f_{3}=0
$$

In this case, $\chi_{D}(\boldsymbol{x})$ is the positive axis of abscissae. It may be represented as $\chi_{D}^{\text {edge }}(\boldsymbol{x})=H\left(x_{1}\right) \delta\left(x_{2}\right)$, where $H\left(x_{1}\right)$ applies to all the positive half plane, and $\delta\left(x_{2}\right)$ only for $x_{2}=0$.

For screw dislocations with Burgers vector parallel to the $x_{1}$ axis, in turn, eqn.19 leads to

$$
f_{1}=B \mu \partial_{x_{3}} \chi_{D}^{\text {screw }}(\boldsymbol{x}), \quad f_{1}=0=f_{2}
$$

where $\partial_{x_{3}} \chi_{D}^{\text {screw }}(\boldsymbol{x})=\mathrm{H}\left(x_{2}\right) \delta^{\prime}\left(x_{3}\right)$.

${ }^{1}$ In the case of elastostatics, the BK theorem reduces to

$$
f_{p}(\boldsymbol{x})=-\int_{S_{\boldsymbol{x}^{\prime}}}\left[u_{i}\right]\left(\boldsymbol{x}^{\prime}\right) \nu_{j} C_{i j p q} \frac{\partial \delta\left(\boldsymbol{x}-\boldsymbol{x}^{\prime}\right)}{\partial x_{q}} \mathrm{~d} S_{\boldsymbol{x}^{\prime}}
$$

2 Note that albeit it generalises the concept of the step function, the characteristic function is not a Heaviside function in higher dimensions: it offers compact support for all points in $D$, not for half spaces. 


\section{BK force representation of cracks}

Employing the same procedure as with dislocations, we are able to provide the force representation of cracks. For brevity's sake, here we shall consider flat cracks subjected to constant loading. In all cases, we shall assume that the crack is thin, which we justify by reference to the fact that in deriving a multipolar field expansion, we are only concerned with the long range fields of the cracks, as these would be relevant in fragmentation or damage evolution studies. Throughout, we assume the crack surface to lie on the $\left(x_{1}, x_{2}\right)$ plane, and the crack plane normal to be the $x_{3}$ plane, and that the crack is subjected to a uniform stress of constant magnitude.

For brevity, in this article we analyse only the cases of the mode I and mode III cracks, omitting the mode II crack as it stands as a converse of the mode I crack.

b. Flat mode I crack. We define a flat mode I crack via its eigenstress. Define the crack as a planar inclusion $D$ of width 0 in the normal to the cracked surface. Its shape may be described via certain planar region $D$ defined, as the dislocations, via the characteristic function

$$
\chi_{D}(\boldsymbol{x})=\left\{\begin{array}{ll}
1 & \boldsymbol{x} \in D \\
0 & \boldsymbol{x} \notin D
\end{array},\right.
$$

the support of which is the locus of points lying on the crack faces.

We consider the case of a mode I crack subjected to a uniform stress of magnitude $\sigma_{33}^{0}$. The corresponding eigenstress $\Sigma_{i j}$ due to the crack is (q.v.[30]):

$$
\Sigma_{33}(\boldsymbol{x})=-\Sigma_{33}^{0} \chi_{D}(\boldsymbol{x}), \quad \Sigma_{32}(\boldsymbol{x})=\Sigma_{31}(\boldsymbol{x})=0 \quad \forall \boldsymbol{x} \in D
$$

The equivalent $\mathrm{BK}$ forces are then given by $f_{j}=-\partial_{i} \Sigma_{i j}[6]$, whereupon

$$
f_{1}(\boldsymbol{x})=0, \quad f_{2}(\boldsymbol{x})=0, \quad f_{3}(\boldsymbol{x})=\sigma_{33}^{0} \partial_{3} \chi_{D}(\boldsymbol{x})
$$

For instance, if $D$ is defined in such a way that

$$
\chi_{D}(\boldsymbol{x})=\left(\mathrm{H}\left(x_{1}\right)-\mathrm{H}\left(x_{1}-L_{1}\right)\right)\left(\mathrm{H}\left(x_{2}\right)-\mathrm{H}\left(x_{2}-L_{2}\right)\right) \delta\left(x_{3}\right),
$$

then

$$
f_{3}(\boldsymbol{x})=\sigma_{33}^{0}\left(\mathrm{H}\left(x_{1}\right)-\mathrm{H}\left(x_{1}-L_{1}\right)\right)\left(\mathrm{H}\left(x_{2}\right)-\mathrm{H}\left(x_{2}-L_{2}\right)\right) \delta^{\prime}\left(x_{3}\right), \quad f_{1}(\boldsymbol{x})=0, \quad f_{2}(\boldsymbol{x})=0,
$$

which defines a $L_{1} \times L_{2}$ wide rectangular crack.

c. Flat mode III crack. As with the mode I cracks, we use $\chi_{D}(\boldsymbol{x})$ to describe the cracked surface. In this case, we assume that the mode I crack is subjected to a uniform shear stress of magnitude $\sigma_{31}^{0}$. The corresponding eigenstress $\Sigma_{i j}$ due to the crack is

$$
\Sigma_{31}(\boldsymbol{x})=-\Sigma_{31}^{0} \chi_{D}(\boldsymbol{x}), \quad \Sigma_{33}(\boldsymbol{x})=\Sigma_{32}(\boldsymbol{x})=0 \quad \forall \boldsymbol{x} \in D
$$

The equivalent BK forces are then given by $f_{j}=-\partial_{i} \Sigma_{i j}$, whereupon

$$
f_{1}(\boldsymbol{x})=-\sigma_{31}^{0} \partial_{3} \chi_{D}(\boldsymbol{x}), \quad f_{2}(\boldsymbol{x})=0, \quad f_{3}(\boldsymbol{x})=-\sigma_{31}^{0} \partial_{1} \chi_{D}(\boldsymbol{x})
$$

\section{FORCE MULTIPOLE EXPANSION OF A DISLOCATION}

In section II we have given the general expresion of the multipolar field expansion of an elastic field. We have seen it to require the description of the defect in terms of its Burridge-Knopoff force representation, which we have derived for both dislocations and cracks in section III. With those two ingredients in place, we now wish to consider the multipolar expansion of a dislocation. From eqn.19 and, in general, section III, we know that dislocations may be regarded as formed by a set of distributed forces acting across the slipped surface.

\section{A. Dipole of screw dislocations}

For illustrative purposes, we shall first consider a dipole of screw dislocations. From eqn.21, we see it is defined by set of force doublets acting across the slipped surface. Here, the dislocation line is placed 
along the $x_{1}$-axis, and the slipped surface defined by its normal along the $x_{3}$ direction; the slipped surface is the positive $x_{1}, x_{2}$ halfplane.

The screw dislocation dipole, with a separation of $L$, is first modelled in antiplane shear as given by

$$
f_{1}\left(x_{1}, x_{2}, x_{3}\right)=B \mu\left[H\left(x_{2}-L\right)-H\left(x_{2}\right)\right] \delta^{\prime}\left(x_{3}\right)
$$

Before proceeding, we note that an indeterminacy arises when integrating $f_{1}$ above over the $x_{1}$ domain, given that the dipole defined above is distributed along the $x_{1}, x_{2}$ plane. We get around it by invoking the linear superposition principle, as follows. First, we study the effect of a line distribution, of the form

$$
f_{1}^{\text {line }}\left(x_{1}, x_{2}, x_{3} ; x_{1}^{\prime}\right)=B \mu\left[H\left(x_{2}-L\right)-H\left(x_{2}\right)\right] \delta^{\prime}\left(x_{3}\right) \delta\left(x_{1}-x_{1}^{\prime}\right) .
$$

Second, we then integrate over the contributions of these lines to eqn. 5 along the $x_{1}^{\prime}$ direction ${ }^{3}$.

In this case, we find that the $0^{\text {th }}$-order moment tensor vanishes as expected. The only non-zero component of the first order dipole moment tensor is

$$
\gamma_{13}^{(1)}=B \mu L
$$

The fact that $\gamma_{13}^{(1)} \neq 0$ entails that a dipole of screw dislocations introduces a torque in the system (in this case acting in the $x_{2}$ direction).

The only non-zero components of the second order, quadrupole moment tensor are

$$
\gamma_{132}^{(2)}=\gamma_{123}^{(2)}=B \mu \frac{L^{2}}{2}
$$

The only non-zero components of the third order, octopole moment tensor are

$$
\gamma_{1322}^{(3)}=\gamma_{1232}^{(3)}=\gamma_{1223}^{(3)}=B \mu \frac{L^{3}}{3}
$$

And so on.

The corresponding displacement fields of these lines in the multipole expansion can be found employing eqn.5. We may use the expression for the 3D elastic Green's function here (see [23]),

$$
G_{i j}(\boldsymbol{x})=\frac{1}{16 \pi \mu(1-\nu) r}\left[(3-4 \nu) \delta_{i j}+\frac{x_{i} x_{j}}{r}\right], \quad r=\sqrt{x_{1}^{2}+x_{2}^{2}+x_{3}^{2}}
$$

Thus, to first order we find

$$
\begin{aligned}
& u_{1}^{\text {line }}(\boldsymbol{x})=-B \mu \lambda \frac{\partial G_{11}(\boldsymbol{x})}{\partial x_{3}}=\frac{B \lambda x_{3}\left((4 \nu-6) x_{1}^{2}+(4 \nu-3)(r)\right)}{16 \pi(\nu-1)\left(x_{1}^{2}+r\right)^{5 / 2}} \\
& u_{2}^{\text {line }}(\boldsymbol{x})=-B \mu \lambda \frac{\partial G_{21}(\boldsymbol{x})}{\partial x_{3}}=\frac{3 B \lambda x_{1} x_{2} x_{3}}{16 \pi(1-\nu)\left(x_{1}^{2}+r\right)^{5 / 2}} \\
& u_{3}^{\text {line }}(\boldsymbol{x})=-B \mu \lambda \frac{\partial G_{31}(\boldsymbol{x})}{\partial x_{3}}=-\frac{B \lambda x_{1}\left(x_{1}^{2}+x_{2}^{2}-2 x_{3}^{2}\right)}{16 \pi(\nu-1)\left(x_{1}^{2}+r\right)^{5 / 2}}
\end{aligned}
$$

Now we integrate out the $x_{1}$ component, to finally obtain the first oder multipole expansion of a dipole of screw dislocations:

$$
u_{1}\left(x_{2}, x_{3}\right)=\frac{B L}{2 \pi} \frac{x_{3}}{x_{2}^{2}+x_{3}^{2}}, \quad u_{2}=0=u_{3}
$$

The expansion may be performed to higher orders using the same method. For instance, the quadrupole expansion of the dipole is

$$
u_{1}\left(x_{2}, x_{3}\right)=-\frac{B L}{2 \pi} \frac{x_{3}}{x_{2}^{2}+x_{3}^{2}}+\frac{B L^{2}}{2 \pi} \frac{x_{2} x_{3}}{\left(x_{2}^{2}+x_{3}^{2}\right)^{2}}, \quad u_{2}=0=u_{3}
$$

\footnotetext{
3 This approach is equivalent to working in the $2 \mathrm{D} x_{2}, x_{3}$ plane with the two dimensional Green's function.
} 
(a) Dipole expansion

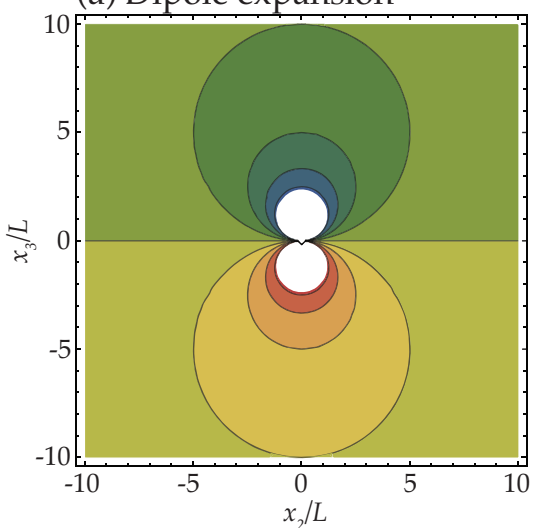

(c) Octopole expansion

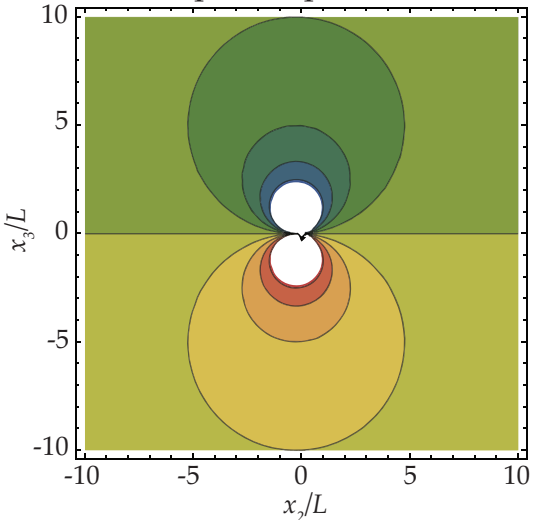

(b) Quadrupole expansion

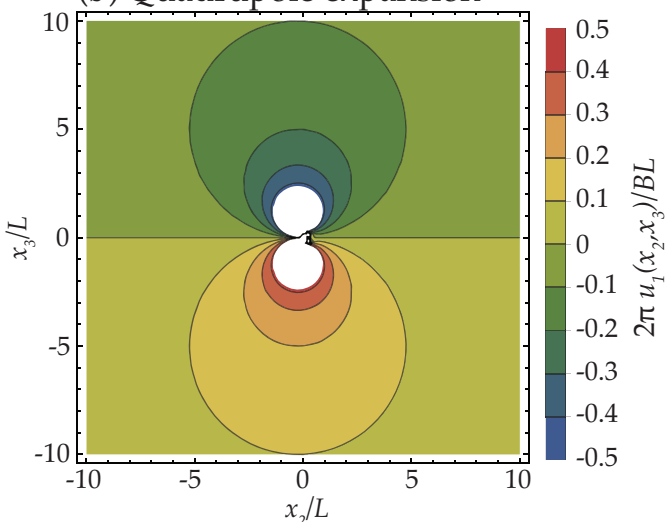

(d) Exact solution

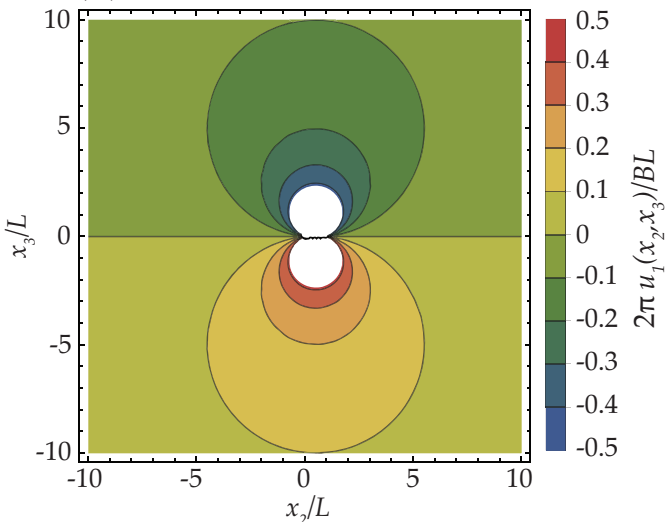

FIG. 1: Comparison between different force multipole expansions of the elastic field of a dipole of screw dislocations.

The octopole expansion, in turn, would render

$$
u_{1}\left(x_{2}, x_{3}\right)=\frac{B L}{2 \pi} \frac{x_{3}}{x_{2}^{2}+x_{3}^{2}}+\frac{B L^{2}}{4 \pi} \frac{x_{2} x_{3}}{\left(x_{2}^{2}+x_{3}^{2}\right)^{2}}+\frac{B L^{3}}{12 \pi} \frac{x_{3}\left(x_{2}^{2}-3 x^{2}\right)}{3\left(x_{2}^{2}+x_{3}^{2}\right)^{3}}, \quad u_{2}=0=u_{3}
$$

It can be shown that this specific multipole expansion corresponds with the Taylor series expansion of the screw dipole's displacement field in series of $L$ about $L=0$. Of course, this was to be expected given that in this case the force distribution we have chosen to represent the screw dislocation dipole was in fact the exact force distribution of Volterra dislocations. Fig.1 provides a comparison between the $u_{1}\left(x_{2}, x_{3}\right)$ fields resulting from each multipolar field expansion and the exact solution.

The quality of this expansion may be judged in fig.1, which compares the exact solution to the dipolar, quadrupolar, and octopolar expansions. Clearly, in the long range the approximation is remarkably good even for the dipole moment expansion alone, even though in the short range increasingly higher order expansions are needed to achieve enough accuracy. From error analysis, we can judge that the dipole expansion falls within $\approx 5 \%$ error or less for distances of $\approx L$. Therefore, as expected, the multipole expansion becomes increasingly accurate for studying the long range behaviour of the dipole of screw dislocations.

\section{MULTIPOLAR FIELD EXPANSION OF A DISLOCATION LOOP}

We shall now consider the multipole expansion of a dislocation loop. In this case, the force representation is given by eqn.19,

$$
f_{p}(\boldsymbol{x})=-C_{i j p q} B_{i} \nu_{j} \frac{\partial \chi_{D}(\boldsymbol{x})}{\partial x_{q}}
$$


The multipole moments are defined with eqn.4, whereupon

$$
\begin{aligned}
\gamma_{p}^{(0)} & =-C_{i j p q} B_{i} \int_{\Omega^{\prime}} \nu_{j} \frac{\partial \chi_{D}\left(\boldsymbol{x}^{\prime}\right)}{\partial x_{q}^{\prime}} \mathrm{d} \Omega^{\prime}=0 \\
\gamma_{p k_{1}}^{(1)} & =-C_{i j p q} B_{i} \nu_{j} \int_{\Omega^{\prime}} x_{k_{1}}^{\prime} \frac{\partial \chi_{D}\left(\boldsymbol{x}^{\prime}\right)}{\partial x_{q}^{\prime}} \mathrm{d} \Omega^{\prime} \\
& =-C_{i j p q} B_{i} \nu_{j} \int_{D^{\prime}} \delta_{k_{1} q} \chi_{D}\left(\boldsymbol{x}^{\prime}\right) \mathrm{d} D^{\prime} \\
& =-C_{i j p q} B_{i} \nu_{j} \delta_{k_{1} q} D
\end{aligned}
$$

where $D$ denotes the area enclosed by the dislocation loop.

$$
\begin{aligned}
\gamma_{p k_{1} k_{2}}^{(2)} & =-C_{i j p q} B_{i} \nu_{j} \int_{\Omega^{\prime}} x_{k_{1}}^{\prime} x_{k_{2}}^{\prime} \frac{\partial \chi_{D}\left(\boldsymbol{x}^{\prime}\right)}{\partial x_{q}^{\prime}} \mathrm{d} \Omega^{\prime} \\
& =-C_{i j p q} B_{i} \nu_{j}\left[\int_{\Omega^{\prime}} \frac{\partial x_{k_{1}}^{\prime}}{\partial x_{q}^{\prime}} x_{k_{2}} \chi_{D}\left(\boldsymbol{x}^{\prime}\right) \mathrm{d} \Omega^{\prime}-\nu_{j} \int_{\Omega^{\prime}} \frac{\partial x_{k_{2}}^{\prime}}{\partial x_{q}^{\prime}} x_{k_{1}} \chi_{D}\left(\boldsymbol{x}^{\prime}\right) \mathrm{d} \Omega^{\prime}\right] \\
& =-C_{i j p q} B_{i} \nu_{j}\left(\delta_{k_{1} q} \delta_{k_{2} u}+\delta_{k_{2} q} \delta_{k_{1} u}\right) J_{u}
\end{aligned}
$$

where $J_{u}$ denotes the first moment of area of the dislocation loop.

One can prove by induction that, in general,

$$
\gamma_{p k_{1} \ldots k_{n}}^{(n)}=-C_{i j p q} B_{i} \nu_{j}\left(\sum_{i=1}^{n} \delta_{k_{i} q} \prod_{j=1, j \neq i}^{n} \delta_{k_{j} u_{\pi_{j ; i}}}\right) J_{u_{1} \ldots u_{n-1}}
$$

where $J_{u_{1} \ldots u_{n-1}}$ denotes the $(n-1)^{t h}$ moment of area of the dislocation loop, and

$$
\pi_{j ; i}= \begin{cases}j, & j<i \\ j-1, & j>i\end{cases}
$$

The resulting displacement field may be obtained using eqn.5.

\section{Multipole expansion of a planar, circular dislocation loop}

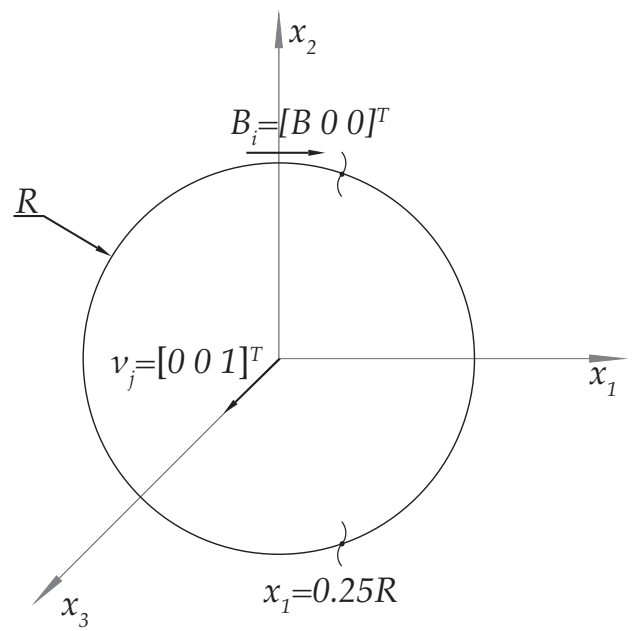

FIG. 2: Schematic of the circular dislocation loop.

For illustrative purposes, here we consider the case of a perfectly circular, planar loop in an isotropic continuum depicted in fig. 2 . The loop is of radius $R$, and centred about the origin of a cartesian coordinate system, with the loop lying in the $x_{1}, x_{2}$ plane, and slip plane normal along the $x_{3}$ direction; we align the Burgers vector with the $x_{1}$ direction. 
(a) Dipole expansion
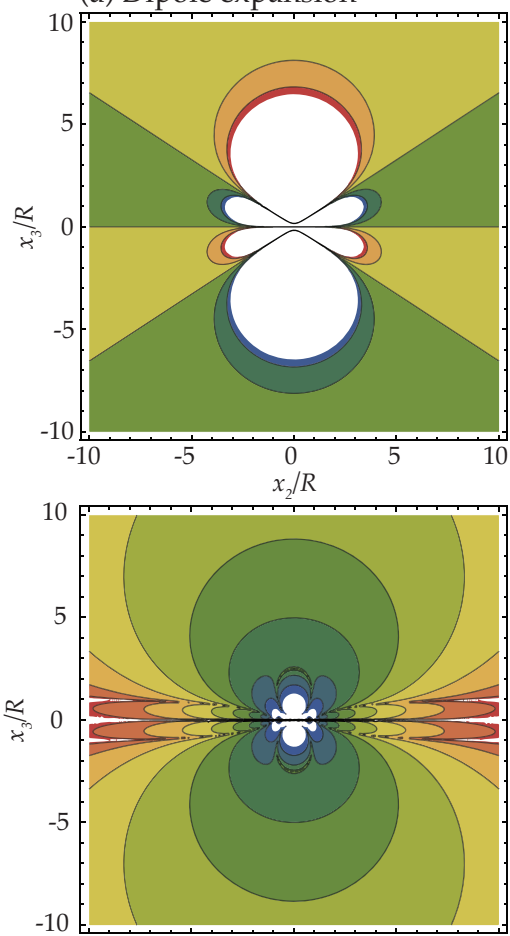

(d) Absolute error field of the dipolar expansion (b) Octopole expansion
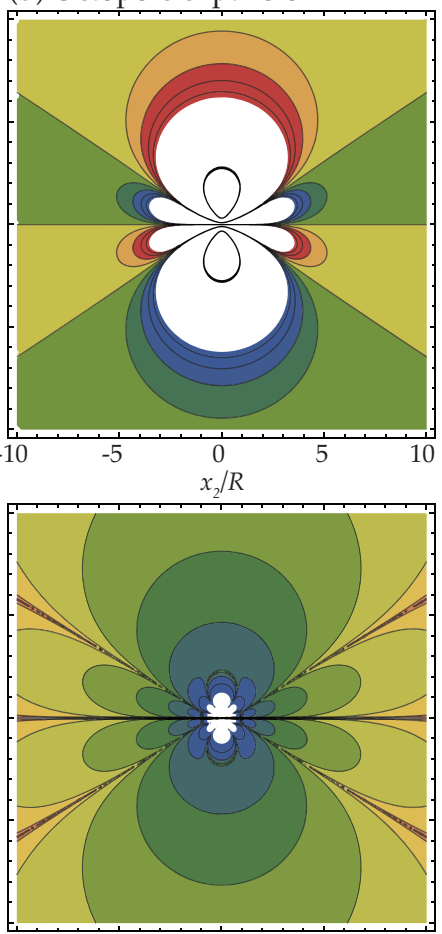

(e) Absolute error field of the octopolar expansion (c) Exact solution
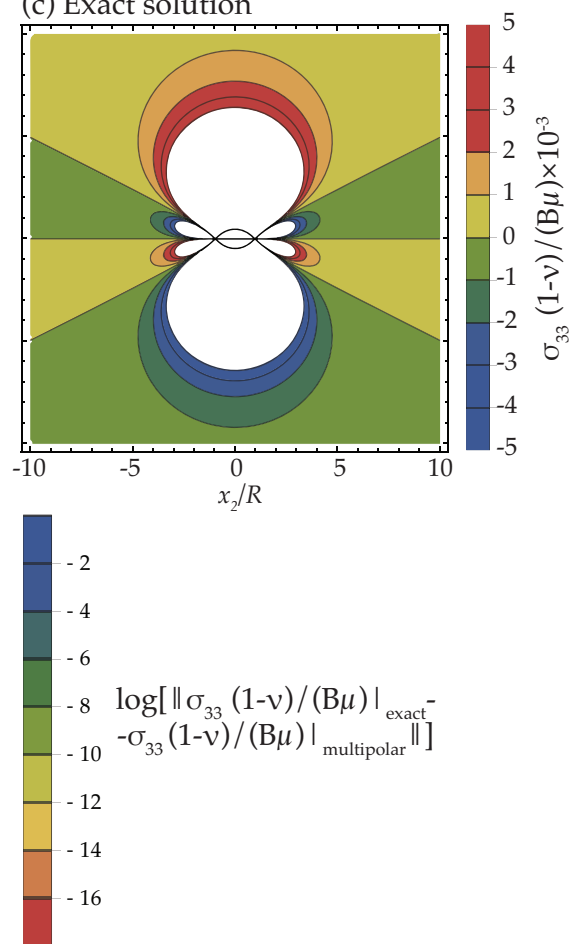

$\log \left[\| \sigma_{33}(1-v) /\left.(\mathrm{B} \mu)\right|_{\text {exact }}\right.$
$\left.-\sigma_{33}(1-v) /\left.(\mathrm{B} \mu)\right|_{\text {multipolar }} \|\right]$

\section{.}

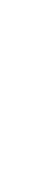

cxact solution of the o

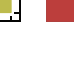

FIG. 3: Dipole and octopole expansion and exact solution of the $\sigma_{33}$ field of a circular dislocation loop.

For brevity, we shall consider the multipole expansion up to third order (dipolar and octopolar moments) alone. The force multipole moments in that case depend on the following moments of area:

$$
D=\pi r, \quad J_{u}=0, \forall u=1,2,3, \quad J_{11}=\frac{\pi r^{4}}{2}=J_{22}, J_{12}=J_{21}=J_{3 u}=J_{u 3}=0, \forall u=1,2,3
$$

And the corresponding non-zero force multipoles are

$$
\begin{array}{r}
\gamma_{13}^{(1)}=\gamma_{31}^{(1)}=B \mu \pi r \\
\gamma_{1311}^{(3)}=\gamma_{1131}^{(3)}=\gamma_{1113}^{(3)}=B \mu \frac{\pi r^{4}}{2} \\
\gamma_{3111}^{(3)}=3 B \mu \frac{\pi r^{4}}{2} \\
\gamma_{1322}^{(3)}=\gamma_{1232}^{(3)}=\gamma_{1223}^{(3)}=B \mu \frac{\pi r^{4}}{2} \\
\gamma_{3122}^{(3)}=\gamma_{3212}^{(3)}=\gamma_{3122}^{(3)}=B \mu \frac{\pi r^{4}}{2}
\end{array}
$$

whereupon the octopolar expansion of the displacement field is

$$
u_{i}=-\left[\frac{\partial G_{i 1}}{\partial x_{3}}+\frac{\partial G_{i 3}}{\partial x_{1}}\right] B \mu \pi r-\frac{1}{3 !}\left[3 \frac{\partial^{3} G_{i 1}}{\partial x_{3} \partial x_{1}^{2}}+3 \frac{\partial^{3} G_{i 3}}{\partial x_{1}^{3}}+3 \frac{\partial^{3} G_{i 1}}{\partial x_{3} \partial x_{2}^{2}}+3 \frac{\partial^{3} G_{i 3}}{\partial x_{1} \partial x_{2}^{2}}\right] B \mu \frac{\pi r^{4}}{2}
$$

where $G_{i j}$ in this case is the three dimensional static isotropic Green's function (see eqn.33 [23]). The resulting field components are given in the Appendix due to their length.

Given the relative simplicity of a circular dislocation loop, we may hope to compare the quality of the multipole expansion with exact (i.e., non expanded) results, which are in fact available (see [31]). Here, the comparison is performed with the stress field components themselves, which may be obtained using $\sigma_{i j}=C_{i j p q} u_{p, q}$. Figure 3, for instance, shows the $\sigma_{33}$ stress component of the dipole and octopole expansions of the said stress field component, alongside the exact solution; both solutions are depicted for a cross section at $x_{1}=0.25 R$. The dipole expansion underestimates $\sigma_{33}$, something which is corrected 
with the octopole expansion; clearly however, the main long-range traits of the exact solution are captured with remarkable accuracy by the dipole expansion.

This was to be expected. Consider eqn.5: the Green's function itself decays as $1 / r$, with $r$ a distance (see [23], although this is clear from eqn.33), so each of its successive derivatives in the multipolar expansion will decay by a factor of $1 / r^{j+1}$, where $j$ is the multipole order of the expansion. That means that the force dipole expansion decays by a factor of $1 / r$, and the octopolar terms by a factor of $1 / r^{4}$, whereby the force dipole moment alone is responsible for the largest contribution to the long range behaviour of the expansion.

The accuracy of the approximation can be increased by expanding the multipole moment expansion to higher orders. Still, the simplicity of this multipole approach alongside its accuracy even for the dipole moment expansion, which depends solely on the area enclosed by the dislocation loop, makes it remarkably simple.

Dislocation interactions are long range, and dislocations move so as to minimise the system's elastic energy (via the Peach-Koehler force)[26]. This multipolar expansion may therefore be employed to estimate with great accuracy the long range dislocation-dislocation interactions and other dislocation mediated long range effects, such as the shielding of cracks or of shock fronts.

\section{MULTIPOLAR FIELD EXPANSION OF CRACKS}

The multipolar field expansions of cracks can be achieved in the same way as for dislocation loops. In section III C we have provided the force representation of flat cracks in modes I, II, and III subjected to uniform stress field components. Using these force representations, we now provide in the following the corresponding multipolar field expansions.

a. Mode I crack. The corresponding force multipole expansion for the mode I crack is then obtained as detailed in the following. The $n$-th order multipolar moment is given by eqn.4, where we substitute $f_{j}$ for eqn.25, leading to:

$$
\begin{aligned}
\gamma_{3 k_{1} \ldots k_{n}}^{(n)} & =\sigma_{33}^{0} \int_{\Omega^{\prime}} x_{k_{1}}^{\prime} \cdot \ldots \cdot x_{k_{n}}^{\prime} \partial_{3} \chi_{D}\left(\boldsymbol{x}^{\prime}\right) \mathrm{d} \Omega \\
& =-\sigma_{33}^{0} \int_{D^{\prime}} \mathrm{d} D^{\prime} \sum_{i=1}^{n} \frac{\partial x_{k_{i}}^{\prime}}{\partial x_{3}^{\prime}} \prod_{j=1, j \neq i}^{n} x_{k_{j}}^{\prime} \\
& =-\sigma_{33}^{0}\left[\sum_{i=1}^{n} \delta_{3 k_{i}} \prod_{j=1, j \neq i}^{n} \delta_{k_{j} u_{\pi_{i, j}}}\right] J_{u_{1} \ldots u_{n-1}}
\end{aligned}
$$

where

$$
J_{u_{1} \ldots u_{n-1}}=\int_{D} x_{u_{1}} \cdot \ldots \cdot x_{u_{n-1}} \mathrm{~d} D
$$

is the $(n-1)^{\text {th }}$ moment of area of the flat crack.

Thus, eqn.48 fully defines the multipolar moments of a mode I flat crack. Combining eqn.48 with eqn.5, we find

$$
u_{i}(\boldsymbol{x})=-\sigma_{33}^{0} \sum_{n=0}^{\infty} \frac{(-1)^{n}}{n !} \frac{\partial^{n} G_{i 3}(\boldsymbol{x})}{\partial x_{k_{1}} \ldots \partial x_{k_{n}}}\left[\sum_{l=1}^{n} \delta_{3 k_{l}} \prod_{j=1, j \neq l}^{n} \delta_{k_{j} u_{\pi_{l, j}}}\right] J_{u_{1} \ldots u_{n-1}}
$$

The corresponding stress field components may be obtained by differentiation of the above.

In particular, the $\sigma_{33}$ component for an isotropic, linear elastic solid is given by $\sigma_{33}=\lambda\left(u_{1,1}+u_{2,2}\right)+$ $(\lambda+2 \mu) u_{3,3}$ :

$$
\begin{aligned}
\sigma_{33}(\boldsymbol{x})=-\sigma_{33}^{0} \sum_{n=0}^{\infty}\left\{\frac{(-1)^{n}}{n !}\left[\sum_{l=1}^{n} \delta_{3 k_{l}} \prod_{j=1, j \neq l}^{n} \delta_{k_{j} u_{\pi_{l, j}}}\right] J_{u_{1} \ldots u_{n-1}} \cdot\right. \\
\left.\cdot\left[\lambda\left(\frac{\partial^{n+1} G_{13}(\boldsymbol{x})}{\partial x_{1} \partial x_{k_{1}} \ldots \partial x_{k_{n}}}+\frac{\partial^{n+1} G_{23}(\boldsymbol{x})}{\partial x_{2} \partial x_{k_{1}} \ldots \partial x_{k_{n}}}\right)+(\lambda+2 \mu)\left(\frac{\partial^{n+1} G_{33}(\boldsymbol{x})}{\partial x_{3} \partial x_{k_{1}} \ldots \partial x_{k_{n}}}\right)\right]\right\}
\end{aligned}
$$

We note that the zero-th order moment, $\gamma_{3}^{(0)}$, will vanish irrespective of the crack's geometry. Hence, the leading order terms are those given by the dipolar moment $\gamma_{3 k_{1}}^{(1)}$, which are directly proportional to the 


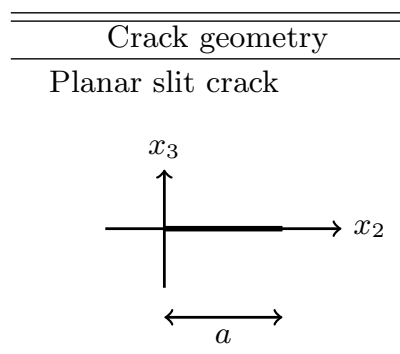

Square crack

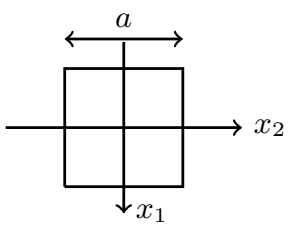

Rectangular crack

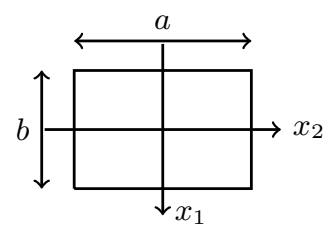

Ellipsoidal crack

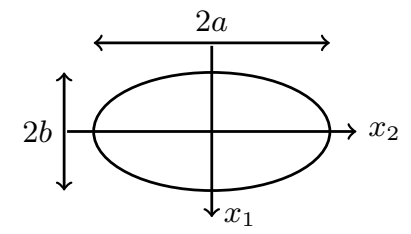

Moments of area

Multipolar moments

$$
\begin{gathered}
J_{0}=0 \\
J_{2}=0, J_{3}=a \\
J_{32}=J_{23}=\frac{a^{2}}{2}, J_{33}=J_{22}=0 \\
J_{223}=J_{322}=J_{232}=-\frac{a^{3}}{3} \\
J_{333}=J_{222}=0 \\
J_{332}=J_{323}=J_{233}=0
\end{gathered}
$$

$$
\begin{gathered}
\gamma_{2}^{(0)}=0 \\
\gamma_{23}^{(1)}=\gamma_{33}^{(1)}=-\sigma_{33}^{0} a \\
\gamma_{332}^{(2)}=\gamma_{223}^{(2)}=-\sigma_{33}^{0} \frac{a^{2}}{2} \\
\gamma_{2322}^{(3)}=\gamma_{2232}^{(3)}=\gamma_{2323}^{(3)}=-\sigma_{33}^{0} \frac{a^{3}}{3}
\end{gathered}
$$

$$
\begin{gathered}
J_{0} \equiv A=a^{2} \\
J_{1}=J_{2}=0 \\
J_{11}=J_{22}=\frac{1}{12} a^{4} \\
J_{12}=0 \\
J_{i 3}=0 \quad \forall i=1,2,3
\end{gathered}
$$

$$
\begin{gathered}
J_{0} \equiv A=a \cdot b \\
J_{1}=J_{2}=0 \\
J_{11}=\frac{1}{12} a^{3} b, J_{22}=\frac{1}{12} b^{3} a \\
J_{12}=0 \\
J_{i 3}=0 \quad \forall i=1,2,3
\end{gathered}
$$

$$
\begin{gathered}
\gamma_{3}^{(0)}=0 \\
\gamma_{31}^{(1)}=\gamma_{32}^{(1)}=-\sigma_{33}^{0} a^{2} \\
\gamma_{3 i j}^{(2)}=0 \quad \forall i, j=1,2,3 \\
\gamma_{3311}^{(3)}=\gamma_{3131}^{(3)}=\gamma_{3113}^{(3)}=-\sigma_{33}^{0} \frac{a^{4}}{12} \\
\gamma_{3322}^{(3)}=\gamma_{3232}^{(3)}=\gamma_{3223}^{(3)}=-\sigma_{33}^{0} \frac{a^{4}}{12}
\end{gathered}
$$

$$
\begin{gathered}
\gamma_{3}^{(0)}=0 \\
\gamma_{31}^{(1)}=\gamma_{32}^{(1)}=-\sigma_{33}^{0} a b \\
\gamma_{3 i j}^{(2)}=0 \quad \forall i, j=1,2,3 \\
\gamma_{3331}^{(3)}=\gamma_{3131}^{(3)}=\gamma_{3113}^{(3)}=-\sigma_{33}^{0} \frac{a^{3} b}{12} \\
\gamma_{3322}^{(3)}=\gamma_{3232}^{(3)}=\gamma_{3223}^{(3)}=-\sigma_{33}^{0} \frac{a b^{3}}{12}
\end{gathered}
$$

$$
\begin{gathered}
\gamma_{3}^{(0)}=0 \\
\gamma_{31}^{(1)}=\gamma_{32}^{(1)}=-\sigma_{33}^{0} \pi a b \\
\gamma_{3 i j}^{(2)}=0 \quad \forall i, j=1,2,3 \\
\gamma_{3311}^{(3)}=\gamma_{3311}^{(3)}=\gamma_{3113}^{(3)}=-\sigma_{33}^{0} \frac{\pi a^{3} b}{4} \\
\gamma_{3322}^{(3)}=\gamma_{3232}^{(3)}=\gamma_{3223}^{(3)}=-\sigma_{33}^{0} \frac{\pi a b^{3}}{4}
\end{gathered}
$$

TABLE I: Force dipole expansion of different flat crack geometries relevant to mode I cracks.

zero-th order moment of area, i.e., the crack's surface area itself. For the isotropic Green's functions, the dipolar term decays with $1 / r$ for $r$ the distance away from the crack (and $1 / r$ for the stress field), and in general the $n^{\text {th }}$ order term will decay with $1 / r^{n}$. Thus, as with dislocations the dipolar term captures the long-range behaviour, and the higher multipolar terms approximate with increasing accuracy the crack's near field.

We note that the multipolar expansion in eqn.50 is entirely general, and applicable to both isotropic and anisotropic materials. We stress that it has been derived from the crack's full elastic field, and not the usual near field about the crack tip. This means that the multipolar expansion will be explicitly dependent on the elastic constants, as is the crack's full field (see [32], p.138; [23],p.261). Given that we are concerned with the far-field behaviour of a crack, this is justified.

The computation of the multipolar moments required in eqn.51 is easily achievable for arbitrary crack geometries, and in many regular geometries they can be tabulated. In table I we collect a number of particular cases.

b. Mode III crack. Similarly to the mode I crack, the mode III crack's multipolar moments are obtained from eqn.4 combined with the corresponding force representation of a mode III crack, in this case given in eqn.27. This entails the following multipolar moments

$$
\begin{aligned}
& \gamma_{1 k_{1} \ldots k_{n}}^{(n)}=\sigma_{33}^{0} \int_{\Omega^{\prime}} x_{k_{1}}^{\prime} \cdot \ldots \cdot x_{k_{n}}^{\prime} \partial_{3} \chi_{D}\left(\boldsymbol{x}^{\prime}\right) \mathrm{d} \Omega \\
& \gamma_{3 k_{1} \ldots k_{n}}^{(n)}=\sigma_{33}^{0} \int_{\Omega^{\prime}} x_{k_{1}}^{\prime} \cdot \ldots \cdot x_{k_{n}}^{\prime} \partial_{1} \chi_{D}\left(\boldsymbol{x}^{\prime}\right) \mathrm{d} \Omega
\end{aligned}
$$


(a) Dipolar expansion

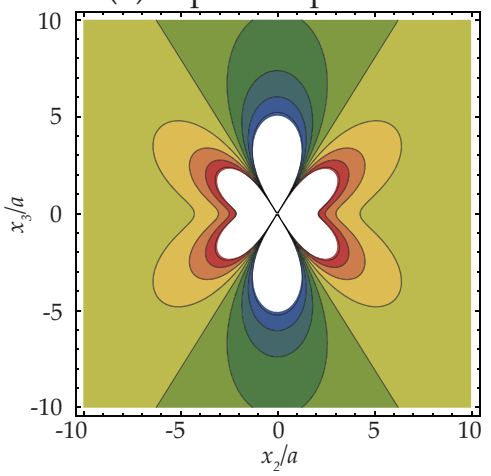

(b) Exact solution

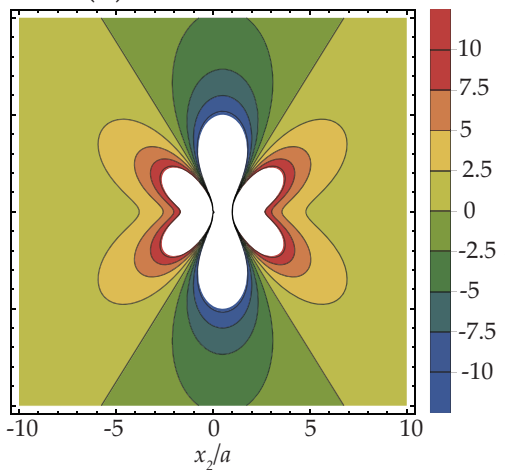

(c) Absolute error field

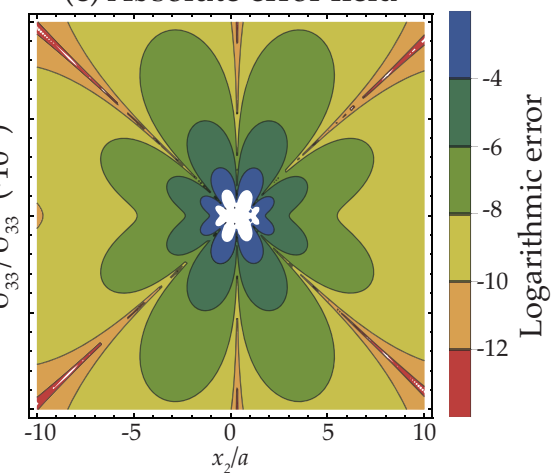

FIG. 4: Dipolar field expansion of a slit crack in 2D, and its comparison to the exact result. Here 'logarithmic error' refers to $\ln \left|\sigma_{33}^{\text {dipole }}-\sigma_{33}^{\text {exact }}\right|-\ln \left[\sigma_{33}^{0}\right]$.

which operating, reduce to

$$
\begin{aligned}
& \gamma_{1 k_{1} \ldots k_{n}}^{(n)}=\sigma_{33}^{0}\left[\sum_{i=1}^{n} \delta_{3 k_{i}} \prod_{j=1, j \neq i}^{n} \delta_{k_{j} u_{\pi_{i, j}}}\right] J_{u_{1} \ldots u_{n-1}} \\
& \gamma_{3 k_{1} \ldots k_{n}}^{(n)}=\sigma_{33}^{0}\left[\sum_{i=1}^{n} \delta_{1 k_{i}} \prod_{j=1, j \neq i}^{n} \delta_{k_{j} u_{\pi_{i, j}}}\right] J_{u_{1} \ldots u_{n-1}}
\end{aligned}
$$

The implied multipolar field expansion is therefore:

$$
u_{i}(\boldsymbol{x})=-\sigma_{33}^{0} \sum_{n=0}^{\infty} \frac{(-1)^{n}}{n !} \frac{\partial^{n} G_{i o}(\boldsymbol{x})}{\partial x_{k_{1}} \ldots \partial x_{k_{n}}}\left[\sum_{l=1}^{n} \delta_{p k_{l}} \prod_{j=1, j \neq l}^{n} \delta_{k_{j} u_{\pi_{l, j}}}\right] J_{u_{1} \ldots u_{n-1}}
$$

where $(o, p)=(1,3)$ or $(3,1)$.

\section{A. Examples}

c. Mode I crack in 2D. We consider first the case of a mode I slit crack in plane stress, subjected to a uniform stress $\sigma_{33}^{0}$. The crack is of length $a$, and we assume it is extended along the $x_{1}$ axis in the $x_{2}, x_{3}$ plane. The corresponding non-zero eigenstress is $\sigma_{33}^{*}\left(x_{2}, x_{3}\right)=\left[\mathrm{H}\left(x_{2}\right)-\mathrm{H}\left(x_{2}-a\right)\right] \delta\left(x_{3}\right) \sigma_{33}^{0}$. In that case, the first two non-zero multipolar moments are given in table I. For example's sake, we focus on the $\sigma_{33}\left(x_{2}, x_{3}\right)$ stress field component; the multipolar field expansion in this case may be attained using eqn.48 whilst integrating $x_{1}$ out of the system, which results in

$\sigma_{33}=-\underbrace{\left[\frac{\left((1-2 \nu) x_{2}^{4}+(2 \nu-3) x_{3}^{4}+6 x_{2}^{2} x_{3}^{2}\right)}{4 \pi(\nu-1) r^{6}}\right] a \sigma_{33}^{0}}_{\text {Dipolar term }}+\underbrace{\left[\frac{\left((2 \nu-1) x_{2}^{5}-2(2 \nu+5) x_{2}^{3} x_{3}^{2}+3(5-2 \nu) x_{2} x_{3}^{4}\right)}{2 \pi(\nu-1) r^{8}}\right] \frac{a^{2} \sigma_{33}^{0}}{2}}_{\text {Quadrupolar term }}$

where $r=x_{2}^{2}+x_{3}^{2}$. As with dislocations, and for the same reasons, the dipolar contribution to the $\sigma_{33}$ field decays with $1 / r$, whilst the quadrupolar with $1 / r^{3}$. We note that these expressions are only valid for the long range field of the crack, and that they are implicitly deduced from the full-field expression of a crack displacement field; as a result, and unlike the crack tip's near fields, both the multipolar expansion and, indeed, the full field of the crack will depend not only on the geometry, but on Poisson's ratio.

The dipolar term is compared with the exact field on fig.4 (which we given in the Appendix), which also offers a measure of the error defined as the logarithm of the absolute value of the difference between the dipolar and exact fields. Similarly to the case of dislocations, we see that even the dipolar expansion's long range fields are accurately captured by the dipolar term alone, albeit as before accuracy may be improved by increasing the multipolar expansion's order. We must stress that the approximation is accurate only for the long range field, and as we see in the fig.4, it does not capture the near field effects. 


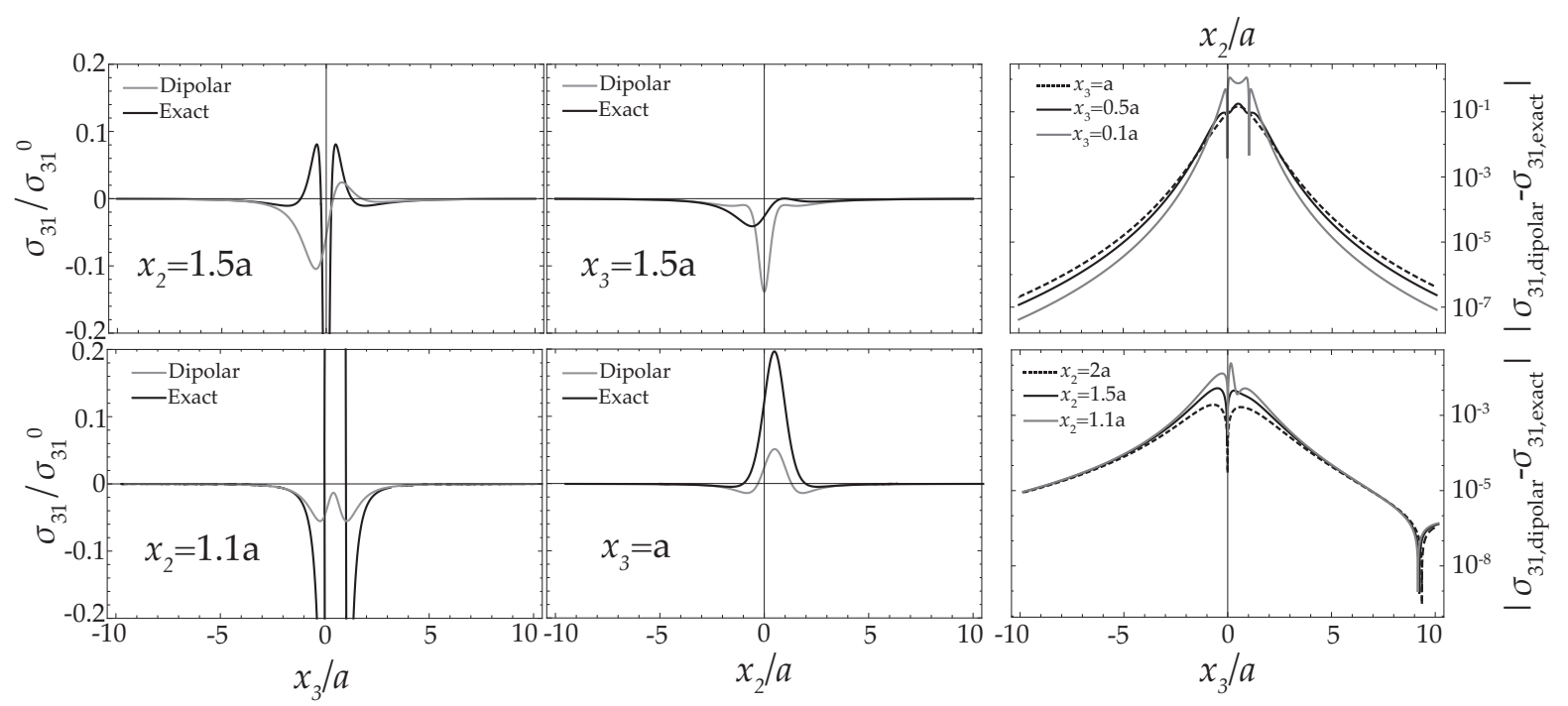

FIG. 5: Values of the $\sigma_{31}$ stress field component of a rectangular crack in the dipolar and exact cases. In this case, $x_{1}=b / 2$, and $b=2 a$. Sections both along the $x_{2}$ and $x_{3}$ directions are provided.

d. Mode III rectangular crack. We consider second the case of a rectangular crack in 3D, such as that described in table I, with $b>a$. Application of eqn.54 rendres the following $\sigma_{13}\left(x_{1}, x_{2}, x_{3}\right)$ multipolar field expansion, to dipolar order:

$$
\sigma_{13}=\left[\frac{\left((\nu+1) x_{1}^{4}+x_{1}^{2}\left((2 \nu-13) x_{3}^{2}-(\nu-2) x_{2}^{2}\right)-\left(x_{2}^{2}+x_{3}^{2}\right)\left((2 \nu-1) x_{2}^{2}-(\nu+1) x_{3}^{2}\right)\right)}{4 \pi(\nu-1) r^{7}}\right] \sigma_{31}^{0} \pi a b
$$

The approximation may be compared with the exact solution, which here we achieved numerically using the finite element method. Here we employed $10^{6}$ regular tetragonal element FE mesh in Fenics [33] of size 0.01 to generate a rectangular, flat crack of size $a=1, b=2$, with Poisson ratio $\nu=0.3$; periodic boundary conditions were employed in a simulation box of size $10 \times 10 \times 10$. We thus compute the stress field, which we compare, for a few slices on the $x_{1}=b / 2$ section about the $x_{2}$ and $x_{3}$ axes with the dipolar approximation, as shown in fig.5. As can be seen, even in 3D the multipolar field expansion is remarkably accurate away from the crack itself.

\section{B. Non-uniform stress expansions}

Given the wide variety of crack geometries and loading combinations involved, the use of a a multipolar expansions for each particular case will require prior assessment of their relative accuracy. Here we have discussed two examples applied to cracks loaded with a uniform, constant remote stress, although many more could be given. That the remote stress is constant and uniform is a standard assumption in the field of fracture (cf.[23, 32]). Nevertheless, in the event the stress field can no longer be assumed to be uniform, the formulation presented here can be readily adapted; all that varies in that case is the value of the multipolar moments themselves.

There is a wide range of different cases to consider in that event, so we shall merely showcase, as an example, the case of the planar mode I crack considered in section VI A subjected to a sinusoidal, i.e., if the force is of the form $f_{3}(\boldsymbol{x})=\sigma_{33}^{0} \sin \left(n \pi x_{2} / a\right)\left[\mathrm{H}\left(x_{2}\right)-\mathrm{H}\left(x_{2}-a\right)\right] \delta^{\prime}\left(x_{3}\right)$ for $n \in \mathbb{N}$ some period. The multipolar moments are readily available, and given by

$\gamma_{3 k_{1} \ldots k_{n}}^{(n)}=\sigma_{33}^{0} \int_{0}^{L} \sin \left(\frac{n \pi x_{2}}{a}\right) \partial_{3}\left[x_{k_{1}} \ldots x_{k_{n}}\right] \mathrm{d} x_{2}=\sigma_{33}^{0}\left[\sum_{i=1}^{n} \delta_{3 k_{i}} \prod_{j=1, j \neq i}^{n} \delta_{k_{j} u_{\pi_{i, j}}}\right] \int_{0}^{L} x_{u_{1}} \ldots x_{u_{n}} \sin \left(\frac{n \pi x_{2}}{a}\right) \mathrm{d} x_{2}$

leading to

$\gamma_{i}^{(0)}=0 \forall i, \quad \gamma_{32}^{(1)}=0, \gamma_{33}^{(1)}=\sigma_{33}^{0} \frac{L(\cos (\pi n)-1)}{\pi n}, \quad \gamma_{332}^{(2)}=\gamma_{323}^{(2)}=\sigma_{33}^{0} \frac{L^{2}(\pi n \cos (\pi n)-\sin (\pi n))}{\pi^{2} n^{2}}, \gamma_{333}^{(2)}=\gamma_{322}^{(2)}=0, \quad \ldots$ 
and the corresponding displacement and stress fields may be computed by eqn.5, the only thing varying being the magnitude of the multipolar moment itself.

\section{ELASTODYNAMIC MULTIPOLAR FIELD EXPANSIONS}

So far, the article has discussed the multipolar field expansions of both dislocations and cracks in an elastostatic continuum. In this section, we extend the multipolar field expansions to a generally elastodynamic setting, where inertial effects are explicitly captured. The crucial force representations of cracks and dislocations require little change. In particular, all one ought to account for is the change in time of the characteristic functions, i.e.,

$$
\chi_{D}(\boldsymbol{x}, t)= \begin{cases}0 & \boldsymbol{x}, t \notin D \\ 1 & \boldsymbol{x}, t \in D\end{cases}
$$

where we use $D(t)$ to denote the support of $\chi_{D}$, and $T$ to denote its temporal support.

In the elastodynamic case, the displacement representation theorem for a force distribution is [34]

$$
u_{i}(\boldsymbol{x}, t)=\int_{-\infty}^{t} \mathrm{~d} t^{\prime} \int_{\Omega^{\prime}} G_{i p}\left(\boldsymbol{x}-\boldsymbol{x}^{\prime}, t-t^{\prime}\right) f_{p}\left(\boldsymbol{x}^{\prime}, t^{\prime}\right) \mathrm{d} \Omega^{\prime}
$$

where $G_{i p}(\boldsymbol{x}, t)$ is in this case the dynamic Green's function (cf.[35]). A priori, there is no formal difference between the elastodynamic and static multipolar field expansion: it suffices to define a space $\Psi=$ $\Omega \times \mathbb{R}_{\text {time }}$, so that now we deal with the collective variable $\psi=\left(x_{1}, x_{2}, x_{3}, t\right)$. However, given the physical distinction between the variables defining the geometry of the defect, and that defining its evolution, it is preferable to establish a distinction between spatial and temporal variables.

If we expand $G_{i p}\left(\boldsymbol{x}-\boldsymbol{x}^{\prime}, t-t^{\prime}\right)$ in Taylor series of $\left(\boldsymbol{x}^{\prime}, t^{\prime}\right)$ about $(0,0)$, we reach the time-space multipolar moments as follows:

$$
G_{i p}\left(\boldsymbol{x}-\boldsymbol{x}^{\prime}, t-t^{\prime}\right)=\sum_{n=0}^{\infty} \frac{1}{n !} \frac{\partial^{n}}{\partial x_{k_{1}} \ldots \partial x_{k_{n}}} \sum_{m=0}^{\infty} \frac{(-1)^{m+n}}{m !} \frac{\partial^{m} G_{i p}(\boldsymbol{x}, t)}{\partial^{m} t} t^{\prime m} x_{k_{1}}^{\prime} \cdot \ldots \cdot x_{k_{n}}^{\prime},
$$

whereupon

$$
u_{i}(\boldsymbol{x}, t)=\sum_{n=0}^{\infty} \frac{1}{n !} \frac{\partial^{n}}{\partial x_{k_{1}} \ldots \partial x_{k_{n}}} \sum_{m=0}^{\infty} \frac{(-1)^{m+n}}{m !} \frac{\partial^{m} G_{i p}(\boldsymbol{x}, t)}{\partial^{m} t} \int_{-\infty}^{t} \mathrm{~d} t^{\prime} \int_{\Omega^{\prime}} t^{\prime m} \cdot x_{k_{1}}^{\prime} \cdot \ldots \cdot x_{k_{n-1}}^{\prime} f_{p}\left(\boldsymbol{x}^{\prime}, t^{\prime}\right) \mathrm{d} \Omega^{\prime}
$$

which defines the dynamic multipolar moment as

$$
\gamma_{p k_{1} \ldots k_{n}}^{(m, n)}=\int_{-\infty}^{t} \mathrm{~d} t^{\prime} \int_{\Omega^{\prime}} t^{\prime m} \cdot x_{k_{1}}^{\prime} \cdot \ldots \cdot x_{k_{n-1}}^{\prime} f_{p}\left(\boldsymbol{x}^{\prime}, t^{\prime}\right) \mathrm{d} \Omega^{\prime}
$$

Thus, as before we reserved the indices $p$ and $\left\{k_{i}\right\}_{i=1}^{\infty}$ to denote spatial variables, and $m$ the temporal order of the multipolar moment. We note that dynamic multipolar moments are causal: the temporal compactness of $\chi_{D}$ ensures that the time integral has finite bounds. The $(m, n)$ order multipolar moments capture the leading terms of the temporal evolution of the defect's field. As such, they are not going to be very accurate: the dynamic Green's function does not decay with increasing $t$, but in fact grows with $t$ (see $[23,35]$ ). Still, they may be useful in capturing the leading term behaviour of a defect's field, particularly if the latter behaves at specific frequencies (cf.[6]).

As an alternative, we may avoid the time series expansion altogether, which renders the displacement field as a time convolution between the spatial multipolar moments and the Green's function's derivatives. Thus if we expand $G_{i p}\left(\boldsymbol{x}-\boldsymbol{x}^{\prime}, t-t^{\prime}\right)$ in Taylor series of $\boldsymbol{x}^{\prime}$ alone, we reach:

$$
G_{i p}\left(\boldsymbol{x}-\boldsymbol{x}^{\prime}, t-t^{\prime}\right)=\sum_{n=0}^{\infty} \frac{(-1)^{n}}{n !} \frac{\partial^{n} G_{i p}\left(\boldsymbol{x}, t-t^{\prime}\right)}{\partial x_{k_{1}} \ldots \partial x_{k_{n}}} x_{k_{1}}^{\prime} \cdot \ldots \cdot x_{k_{n}}^{\prime},
$$

so that the displacement field

$$
\begin{aligned}
u_{i}(\boldsymbol{x}, t) & =\sum_{n=0}^{\infty} \frac{(-1)^{n}}{n !} \int_{-\infty}^{t} \mathrm{~d} t^{\prime} \frac{\partial^{n} G_{i p}\left(\boldsymbol{x}, t-t^{\prime}\right)}{\partial x_{k_{1}} \ldots \partial x_{k_{n}}} \int_{\Omega^{\prime}} x_{k_{1}}^{\prime} \cdot \ldots \cdot x_{k_{n-1}}^{\prime} f_{p}\left(\boldsymbol{x}^{\prime}, t^{\prime}\right) \mathrm{d} \Omega^{\prime} \\
& =\sum_{n=0}^{\infty} \frac{(-1)^{n}}{n !} \int_{-\infty}^{t} \mathrm{~d} t^{\prime} \frac{\partial^{n} G_{i p}\left(\boldsymbol{x}, t-t^{\prime}\right)}{\partial x_{k_{1}} \ldots \partial x_{k_{n}}} \gamma_{k_{1} \ldots k_{n}}^{(n)}\left(t^{\prime}\right)=\sum_{n=0}^{\infty} \frac{(-1)^{n}}{n !} \frac{\partial^{n}\left[G_{i p} * \gamma_{p k_{1} \ldots k_{n}}^{(n)}\right]}{\partial x_{k_{1}} \ldots \partial x_{k_{n}}}
\end{aligned}
$$


where

$$
\gamma_{k_{1} \ldots k_{n}}^{(n)}\left(t^{\prime}\right)=\int_{\Omega^{\prime}} x_{k_{1}}^{\prime} \cdot \ldots \cdot x_{k_{n-1}}^{\prime} f_{p}\left(\boldsymbol{x}^{\prime}, t^{\prime}\right) \mathrm{d} \Omega^{\prime}
$$

is the spatial multipolar moments, now a function of time. Although this requires solving the $G_{i p} * \gamma_{p k_{1} \ldots k_{n}}^{(n)}$ time convolution, which at higher orders may not be analytically accessible, we achieve a far more accurate description of the long-range temporal behaviour of the defect, and given that in general the low order spatial multipolar moments suffice in the description of their long range fields, this alternative description of the multipolar moment field may suffice in many circumstances. In the following we explore and compare the two situations.

\section{A. Dynamic multipolar expansion of dislocations}

\section{Dynamic force representations.}

The crucial force representations of cracks and dislocations require little change. In particular, all one ought to account for is the change in time of the characteristic functions, i.e.,

$$
\chi_{D}(\boldsymbol{x}, t)= \begin{cases}0 & \boldsymbol{x}, t \notin D \\ 1 & \boldsymbol{x}, t \in D\end{cases}
$$

Thus, in this case the dynamic force representation of a dislocation loop will be

$$
f_{p}(\boldsymbol{x}, t)=-C_{i j p q} B_{i} \nu_{j} \frac{\partial \chi_{D}(\boldsymbol{x}, t)}{\partial x_{q}}
$$

Clearly, $\chi_{D}(\boldsymbol{x}, t)$ stores the 'shape' history of the dislocation loop, i.e., the shape the loop has had since $t=0$. It generalises the past history function that records the past positions of straight dislocations lines, and which is crucial in describing the dynamic fields of dislocations [36, 37]. Indeed, for the case of straight dislocations, the force representation can be given by defining some past history function $x=\eta(t)$, which describes the position of the straight dislocation. If the screw dislocation moves with such past history, then the force representation would merely be

$$
f_{3}\left(x_{2}, x_{3}\right)=B \mu \mathrm{H}\left(x_{2}-\eta(t)\right) \delta\left(x_{3}\right) \mathrm{H}(t), \quad f_{1}=0=f_{2}
$$

The corresponding force representation for the edge dislocation would be

$$
f_{1}=0, \quad f_{2}\left(x_{2}, x_{3}\right)=B \mu \mathrm{H}\left(x_{2}-\eta(t)\right) \delta^{\prime}\left(x_{3}\right) \mathrm{H}(t), \quad f_{3}\left(x_{2}, x_{3}\right)=B \mu \delta\left(x_{2}-\eta(t)\right) \delta\left(x_{3}\right) \mathrm{H}(t)
$$

\section{Multipolar expansion}

We consider the general case of a dislocation loop. In this event, the multipolar moments are

$$
\begin{aligned}
\gamma_{p k_{1} \ldots k_{n}}^{(m, n)} & =-C_{i j p q} B_{i} \nu_{j} \int_{\mathbb{R}} \mathrm{d} t \int_{\Omega} t^{m} \cdot x_{k_{1}} \cdot \ldots \cdot x_{k_{n}} \frac{\partial \chi_{D}(\boldsymbol{x}, t)}{\partial x_{q}} \mathrm{~d} \Omega \\
& =-C_{i j p q} B_{i} \nu_{j} \int_{T} \mathrm{~d} t t^{m}\left(\sum_{i=1}^{n} \delta_{k_{i} q} \prod_{j=1, j \neq i}^{n} \delta_{k_{j} u_{\pi_{j ; i}}}\right) \int_{D(t)} x_{u_{1}} \cdot \ldots \cdot x_{u_{n-1}} \mathrm{~d} D(t) \\
& =-C_{i j p q} B_{i} \nu_{j}\left(\sum_{i=1}^{n} \delta_{k_{i} q} \prod_{j=1, j \neq i}^{n} \delta_{k_{j} u_{\pi_{j ; i}}}\right) \int_{T} J_{u_{1} \ldots u_{n-1}}(t) t^{m} \mathrm{~d} t
\end{aligned}
$$

where $T$ denotes the temporal support of $\chi_{D}(\boldsymbol{x}, t)$, and where

$$
J_{u_{1} \ldots u_{n-1}}(t)=\int_{D(t)} x_{u_{1}} \cdot \ldots \cdot x_{u_{n-1}} \mathrm{~d} D(t)
$$

is the instantaneous $(n-1)^{\text {th }}$ moment of area of the loop, over which one must integrate with increasing powers of $t$ to compute the $(m, n)^{\text {th }}$ order multipolar moment. The spatial multipolar moments take the same form as in eqn.43. 


\section{Examples}

Let us consider the case of an injected, quiescent dipole of edge dislocations: at time $t=0$, the dipole of distance $L$ is suddenly created. The corresponding force representation would then be

$$
f_{2}\left(x_{2}, x_{3}\right)=B \mu\left[\mathrm{H}\left(x_{2}\right)-\mathrm{H}\left(x_{2}-L\right)\right] \delta^{\prime}\left(x_{3}\right) \mathrm{H}(t), \quad f_{3}\left(x_{2}, x_{3}\right)=B \mu\left[\delta\left(x_{2}\right)-\delta\left(x_{2}-L\right)\right] \delta\left(x_{3}\right) \mathrm{H}(t)
$$

Given the plane strain conditions, we may restrict ourselves to the $\left(x_{2}, x_{3}\right)$ plane, and employ the $2 \mathrm{D}$ version of the dynamic Green's function (see [35], p.412):

$$
\begin{aligned}
G_{i p}=\frac{1}{2 \pi \rho}\left[\left(\frac{x_{i} x_{p}}{r^{4}} \frac{2 t^{2}-a^{2} r}{\sqrt{t^{2}-a^{2} r}}-\delta_{i p}\right.\right. & \left.\frac{\sqrt{t^{2}-a^{2} r}}{r}\right) \mathrm{H}(t-a r)+ \\
& \left.+\left(\delta_{i p} \frac{\sqrt{t^{2}-b^{2} r}}{r}+\delta_{i p} \frac{b^{2}}{\sqrt{t^{2}-b^{2} r}}-\frac{x_{i} x_{p}}{r^{4}} \frac{2 t^{2}-b^{2} r}{\sqrt{t^{2}-b^{2} r}}\right) \mathrm{H}(t-b r)\right]
\end{aligned}
$$

where $\rho$ is the medium's density, $a=1 / c_{l}$ and $b=1 / c_{t}$ the longitudinal and transverse slownesses of sound (and $c_{l}$ and $c_{t}$ the longitudinal and transverse speeds of sound), and $r=\sqrt{x_{2}^{2}+x_{3}^{2}}$.

In this case, the $0^{\text {th }}$ order moments vanish, and the non-zero multipolar moments are

$$
\gamma_{32}^{(m, 1)}=\gamma_{23}^{(m, 1)}=B \mu L \frac{t^{m+1}}{m+1}, \quad \gamma_{322}^{(m, 2)}=\gamma_{232}^{(m, 2)}=\gamma_{223}^{(m, 2)}=B \mu \frac{L^{2}}{2} \frac{t^{m+1}}{m+1}, \quad \ldots
$$

Having established in the static case that the dipolar approximation is a reasonable approximation of the long range behaviour of dislocations here, for comparison's sake, we will consider the $m=0$ and $m=1$ temporal moments expanded up to dipolar order. By differentiation of the displacement field components $\sigma_{23}$ stress field component may be obtained from differentiation of the displacement fields as given by

$$
\begin{aligned}
& \sigma_{23}^{(0,1)}=\frac{B L \mu t}{2 \pi b^{2} r^{8}}\left(\frac { \mathrm { H } ( t - b r ) } { ( t ^ { 2 } - b ^ { 2 } r ) ^ { 5 / 2 } } \left[-3 b^{6} r^{6}\left(x_{2}^{4}+4 x_{2}^{3} x_{3}-8 x_{2}^{2} x_{3}^{2}-6 x_{2} x_{3}^{3}+x_{3}^{4}\right)+\right.\right. \\
& +12 b^{4} r^{4} t^{2}\left(2 x_{2}^{4}+5 x_{2}^{3} x_{3}-11 x_{2}^{2} x_{3}^{2}-10 x_{2} x_{3}^{3}+2 x_{3}^{4}\right)-10 b^{2} t^{4}\left(3 x_{2}^{6}+8 x_{2}^{5} x_{3}-15 x_{2}^{4} x_{3}^{2}-8 x_{2}^{3} x_{3}^{3}-15 x_{2}^{2} x_{3}^{4}-16 x_{2} x_{3}^{5}+\right. \\
& \left.\left.+3 x_{3}^{6}\right)+4 t^{6}\left(3 x_{2}^{4}+8 x_{2}^{3} x_{3}-18 x_{2}^{2} x_{3}^{2}-16 x_{2} x_{3}^{3}+3 x_{3}^{4}\right)\right]-\frac{2 \mathrm{H}(t-a r)}{\left(t^{2}-a^{2} r\right)^{5 / 2}}\left[-a^{6} r^{6}\left(2 x_{2}^{4}+6 x_{2}^{3} x_{3}-11 x_{2}^{2} x_{3}^{2}-9 x_{2} x_{3}^{3}+2 x_{3}^{4}\right)+\right. \\
& +a^{4} r^{4} t^{2}\left(11 x_{2}^{4}+30 x_{2}^{3} x_{3}-68 x_{2}^{2} x_{3}^{2}-60 x_{2} x_{3}^{3}+11 x_{3}^{4}\right)-5 a^{2} t^{4}\left(3 x_{2}^{6}+8 x_{2}^{5} x_{3}-15 x_{2}^{4} x_{3}^{2}-8 x_{2}^{3} x_{3}^{3}-\right. \\
& \left.\left.-15 x_{2}^{2} x_{3}^{4}-16 x_{2} x_{3}^{5}+3 x_{3}^{6}\right)+2 t^{6}\left(3 x_{2}^{4}+8 x_{2}^{3} x_{3}-18 x_{2}^{2} x_{3}^{2}-16 x_{2} x_{3}^{3}+3 x_{3}^{4}\right]\right) \\
& \sigma_{23}^{(1,1)}=\frac{B L \mu t}{4 \pi b^{2} r^{8}}\left(\frac { \mathrm { H } ( t - b r ) } { ( t ^ { 2 } - b ^ { 2 } r ) ^ { 7 / 2 } } \left[6 b^{8} r^{8}\left(x_{2}^{4}+4 x_{2}^{3} x_{3}-8 x_{2}^{2} x_{3}^{2}-6 x_{2} x_{3}^{3}+x_{3}^{4}\right)-\right.\right. \\
& -21 b^{6} r^{6} t^{2}\left(x_{2}^{4}+4 x_{2}^{3} x_{3}-8 x_{2}^{2} x_{3}^{2}-6 x_{2} x_{3}^{3}+x_{3}^{4}\right)+20 b^{4} r^{4} t^{4}\left(3 x_{2}^{4}+7 x_{2}^{3} x_{3}-15 x_{2}^{2} x_{3}^{2}-14 x_{2} x_{3}^{3}+3 x_{3}^{4}\right)- \\
& \left.-14 b^{2} t^{6}\left(3 x_{2}^{6}+8 x_{2}^{5} x_{3}-15 x_{2}^{4} x_{3}^{2}-8 x_{2}^{3} x_{3}^{3}-15 x_{2}^{2} x_{3}^{4}-16 x_{2} x_{3}^{5}+3 x_{3}^{6}\right)+4 t^{8}\left(3 x_{2}^{4}+8 x_{2}^{3} x_{3}-18 x_{2}^{2} x_{3}^{2}-16 x_{2} x_{3}^{3}+3 x_{3}^{4}\right)\right]- \\
& -\frac{2 \mathrm{H}(t-a r)}{\left(t^{2}-a^{2} r\right)^{7 / 2}}\left[2 a^{8} r^{8}\left(2 x_{2}^{4}+6 x_{2}^{3} x_{3}-11 x_{2}^{2} x_{3}^{2}-9 x_{2} x_{3}^{3}+2 x_{3}^{4}\right)-7 a^{6} r^{6} t^{2}\left(2 x_{2}^{4}+6 x_{2}^{3} x_{3}-11 x_{2}^{2} x_{3}^{2}-9 x_{2} x_{3}^{3}+2 x_{3}^{4}\right)+\right. \\
& +5 a^{4} r^{4} t^{4}\left(5 x_{2}^{4}+14 x_{2}^{3} x_{3}-32 x_{2}^{2} x_{3}^{2}-28 x_{2} x_{3}^{3}+5 x_{3}^{4}\right)-7 a^{2} t^{6}\left(3 x_{2}^{6}+8 x_{2}^{5} x_{3}-15 x_{2}^{4} x_{3}^{2}-8 x_{2}^{3} x_{3}^{3}-15 x_{2}^{2} x_{3}^{4}-\right. \\
& \left.\left.\left.-16 x_{2} x_{3}^{5}+3 x_{3}^{6}\right)+2 t^{8}\left(3 x_{2}^{4}+8 x_{2}^{3} x_{3}-18 x_{2}^{2} x_{3}^{2}-16 x_{2} x_{3}^{3}+3 x_{3}^{4}\right)\right]\right)
\end{aligned}
$$

Alternatively, the spatial multipolar moments are simply

$$
\gamma_{32}^{(1)}(t)=\gamma_{32}^{(1)}(t)=B \mu L \mathrm{H}(t), \quad \gamma_{322}^{(2)}(t)=\gamma_{232}^{(2)}(t)=\gamma_{223}^{(2)}(t)=B \mu \frac{L^{2}}{2} \mathrm{H}(t), \quad \ldots
$$

Which render a (in this case) simpler multipolar field expansion, because they entail no time convolution. In this case, we obtain:

$$
\begin{array}{r}
\sigma_{23}^{(1)}=\frac{B \mu L t}{2 \pi b^{2} r^{8}}\left(\frac { 4 \mathrm { H } ( t - a r ) } { ( t ^ { 2 } - a ^ { 2 } r ^ { 2 } ) ^ { 3 / 2 } } \left(a^{4} r^{4}\left(2 x_{2}^{4}-11 x_{2}^{2} x_{3}^{2}+2 x_{3}^{4}\right)-5 a^{2} t^{2}\left(x_{2}^{6}-5 x_{2}^{4} x_{3}^{2}-5 x_{2}^{2} x_{3}^{4}+x_{3}^{6}\right)+\right.\right. \\
\left.+3 t^{4}\left(x_{2}^{4}-6 x_{2}^{2} x_{3}^{2}+x_{3}^{4}\right)\right)-\frac{\mathrm{H}(t-b r)}{\left(t^{2}-b^{2} r^{2}\right)^{3 / 2}}\left(b^{4} r^{4}\left(7 x_{2}^{4}-46 x_{2}^{2} x_{3}^{2}+7 x_{3}^{4}\right)-\right. \\
\left.\left.-20 b^{2} t^{2}\left(x_{2}^{6}-5 x_{2}^{4} x_{3}^{2}-5 x_{2}^{2} x_{3}^{4}+x_{3}^{6}\right)+12 t^{4}\left(x_{2}^{4}-6 x_{2}^{2} x_{3}^{2}+x_{3}^{4}\right)\right)\right)
\end{array}
$$



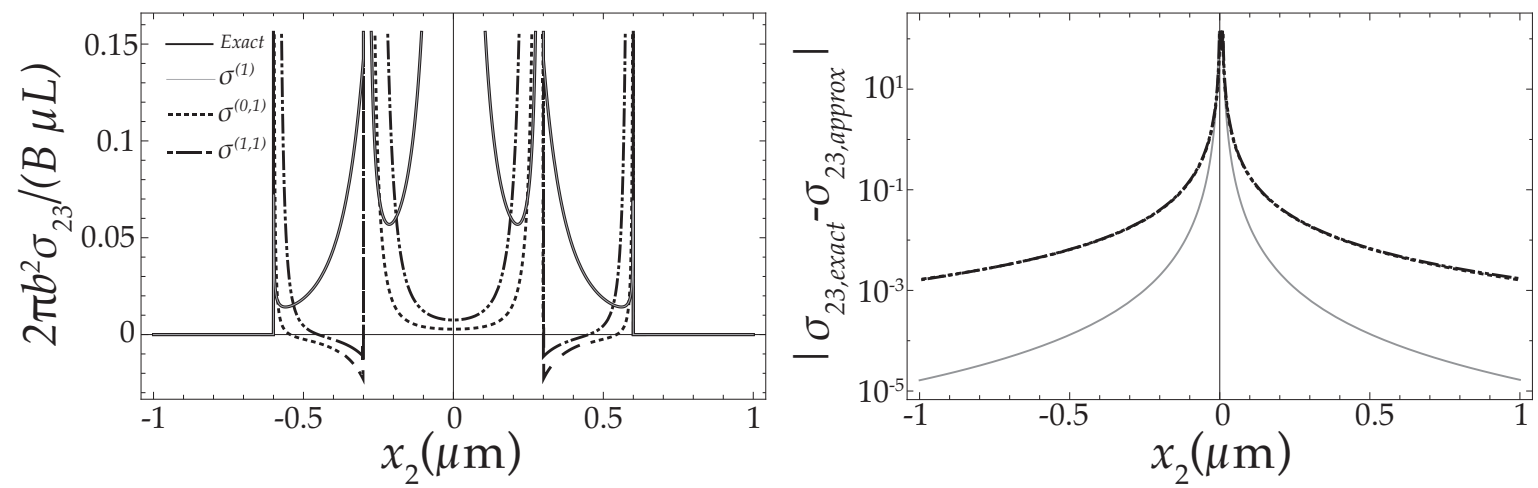

FIG. 6: $\sigma_{23}$ component of stress along $x_{3}=0$ for the spatial and temporal dipolar fields of a quiescent dipole of edge dislocations. Here $a=1 / 6000, b=1 / 3000, L=10^{-2} \mu \mathrm{m}, t=0.1 \mathrm{~ns}$.
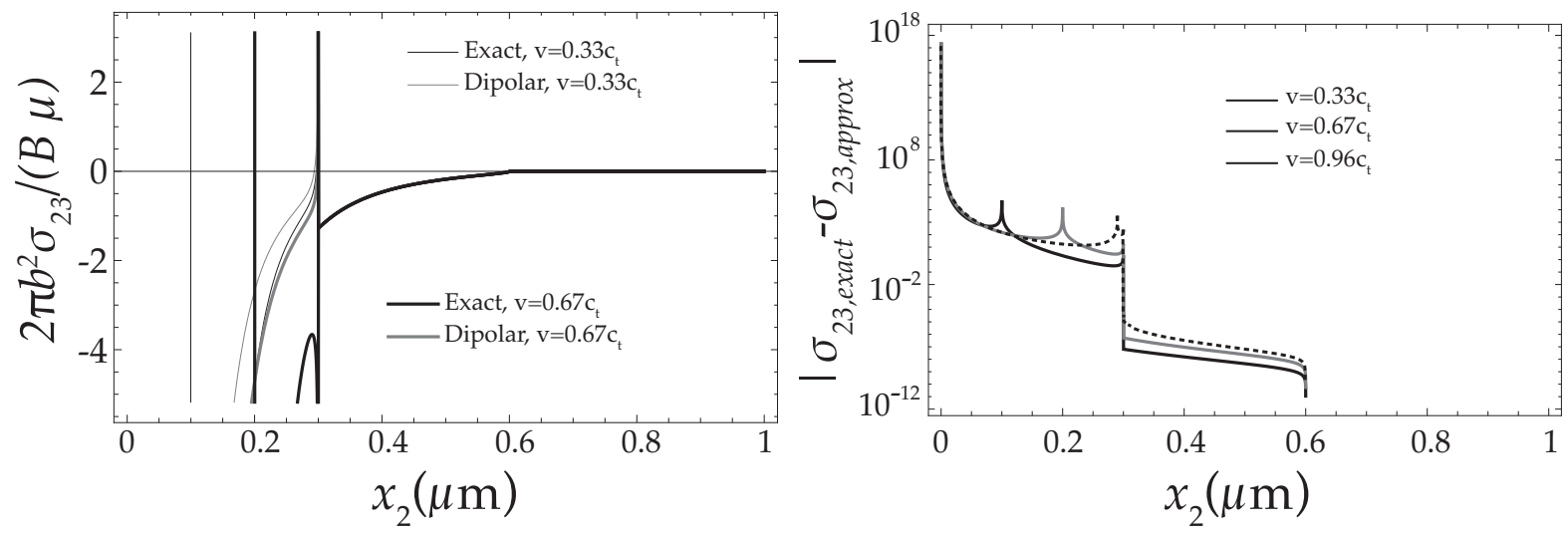

FIG. 7: $\sigma_{23}$ component of stress along $x_{3}=0$ for the spatial dipolar field of a moving dipole of edge dislocations at different speeds, and the associated absolute error field. Here $a=1 / 6000, b=1 / 3000$,

$$
L=10^{-2} \mu \mathrm{m}, t=0.1 \mathrm{~ns} \text {. }
$$

In fig.6, we compare the different multipolar field expansions (both temporal and spatial) with the exact solution derived by Gurrutxaga-Lerma et al.[36]. It is clear that the spatial multipolar expansion, involving the convolution of the characteristic function with the Green's function, is extremely accurate to dipolar term, whereas the temporal multipolar ones only capture correctly the order of the singularities at the wavefronts; the error entailed by the later remains of the same order of magnitude.

Clearly, the time-convolution solution should be the preferred one when accuracy is sought after, so long as the time convolution may be achieved. For instance, we may consider the case where the dislocation on the right hand side of the dipole moves with constant speed $v=1 / d$ away from the one on the left hand side, so that now $L=v t$. In that case, the spatial multipolar moments are

$$
\gamma_{32}^{(1)}(t)=\gamma_{32}^{(1)}(t)=B \mu v t \mathrm{H}(t), \quad \gamma_{322}^{(2)}(t)=\gamma_{232}^{(2)}(t)=\gamma_{223}^{(2)}(t)=B \mu \frac{(v t)^{2}}{2} \mathrm{H}(t), \quad \ldots
$$

and we are forced to compute the convolution in time in eqn.63 explicitly. The resulting $\sigma_{23}$ field may then be computed by differentiation; due to the length of the full field approximation, here we reproduce the briefer component for $x_{3}=0$ (i.e., along the dipole's slip direction) to dipolar order:

$$
\sigma_{23}^{(1)}=\frac{4 t(t v-1) \sqrt{t^{2}-a^{2} x_{2}^{2}}}{x_{2}^{3}} \mathrm{H}\left(t-a x_{2}\right)-\frac{\left(b^{2} x_{2}^{2}-2 t^{2}\right)^{2}\left(t v-x_{2}\right)}{t x_{2}^{4} \sqrt{t^{2}-b^{2} x_{2}^{2}}} \mathrm{H}\left(t-b x_{2}\right)
$$

We compare this approximation to that given by the exact solution (see [36, 38]) in fig.7. As can be seen, the dipolar approximation is accurate for the longitudinal wave components (i.e., $x_{2}>0.3$ ), but becomes increasingly inaccurate for the transverse wave components as one increases the dislocation's speed relative to the transverse speed of sound, $c_{t}$.

This highlights the dual problematic posed by the dynamic multipolar field expansion when the dislocation (or any other defect) is moving. On the one hand, the multipolar expansion subsumes the whole 
force distribution to a single force multipole centred at the origin: as the dislocation moves away from the origin, the multipolar approximation becomes increasingly unable to capture such displacement, and therefore increasingly inaccurate about the origin. On the other hand, the fields of a dislocation experience a dynamic magnification ahead of the core [37, 39]; since the dipolar expansion does not move, it does not capture such magnification. The result in the case of dislocations (and arguably, of cracks), is that as their speed increases towards the transverse speed of sound, the multipolar expansion becomes increasingly inaccurate for the transverse field component; this can be seen in fig.7 for $x_{2} \in(0.2,0.3)$. The far field, in this case represented by the longitudinal field, is invariably going to be correctly captured, since the dislocation's speed will generally not be able to surmount the transverse speed barrier, which occurs at about $50 \%$ of $c_{l}$ in most materials. This suggests that the longitudinal field of dislocations, be them planar or three dimensional loops, will be accurately captured by their multipolar expansion inasmuch as the dislocation segments will be limited to gliding at speeds below the transverse speed of sound.

These problems and general guidelines (i.e., that the multipolar field expansion is generally very accurate for the longitudinal field components, but less so for transverse wave components) extends to the case of three dimensional dislocation loops. Due to the length and complexity of this case, it is omitted from the current discussion and will be the focus of future work. The problems posed by the dynamic multipolar field expansion does not demerit their use in capturing, at a fraction of the computational cost, the far field behaviour of the dynamic dislocation.

\section{B. Dynamic cracks}

The force representation of dynamic cracks stands in direct analogy to the static case given in section III C. Thus, for a mode I crack:

$$
f_{1}(\boldsymbol{x}, t)=0, \quad f_{2}(\boldsymbol{x}, t)=0, \quad f_{3}(\boldsymbol{x}, t)=\sigma_{33}^{0}(t) \partial_{3} \chi_{D}(\boldsymbol{x}, t)
$$

and for a mode III crack:

$$
f_{1}(\boldsymbol{x}, t)=-\sigma_{31}^{0}(t) \partial_{3} \chi_{D}(\boldsymbol{x}, t), \quad f_{2}(\boldsymbol{x}, t)=0, \quad f_{3}(\boldsymbol{x}, t)=-\sigma_{31}^{0}(t) \partial_{1} \chi_{D}(\boldsymbol{x}, t)
$$

The corresponding multipolar field expansions may be obtained as done in the static case.

a. Mode I cracks. In this case, the spatio-temporal multipolar moments requiring specifying the time form of the applied load. We assume that $\sigma_{33}^{0}$ is uniform in space, and evolves in time in such a way that $\sigma_{33}^{0}(t)=\sigma_{33}^{0} f(t)$, where $f(t)$ captures its time evolution, and $\sigma_{33}^{0}$ defines its magnitude. Then, using eqn.61:

$$
\begin{aligned}
\gamma_{3 k_{1} \ldots k_{n}}^{(m, n)} & =\sigma_{33}^{0} \int_{-\infty}^{t} \mathrm{~d} t^{\prime} t^{\prime m} \int_{\Omega^{\prime}} x_{k_{1}}^{\prime} \cdot \ldots \cdot x_{k_{n}}^{\prime} \partial_{3} \chi_{D}\left(\boldsymbol{x}^{\prime}, t^{\prime}\right) f\left(t^{\prime}\right) \mathrm{d} \Omega \\
& =-\sigma_{33}^{0} \int_{-\infty}^{t} \mathrm{~d} t^{\prime} t^{\prime m} f\left(t^{\prime}\right) \int_{D^{\prime}\left(t^{\prime}\right)} \mathrm{d} D^{\prime}\left(t^{\prime}\right) \sum_{i=1}^{n} \frac{\partial x_{k_{i}}^{\prime}}{\partial x_{3}^{\prime}} \prod_{j=1, j \neq i}^{n} x_{k_{j}}^{\prime} \\
& =-\sigma_{33}^{0}\left[\sum_{i=1}^{n} \delta_{3 k_{i}} \prod_{j=1, j \neq i}^{n} \delta_{k_{j} u_{\pi_{i, j}}}\right] \int_{-\infty}^{t} t^{\prime m} J_{u_{1} \ldots u_{n-1}}\left(t^{\prime}\right) f\left(t^{\prime}\right) \mathrm{d} t^{\prime}
\end{aligned}
$$

The spatial force multipoles are given by eqn.48.

b. Mode III cracks. In this case, the spatio-temporal multipolar moments requiring specifying the time form of the applied load. As for mode I, we assume that $\sigma_{31}^{0}(t)$ is uniform in space, and evolves in time in such a way that $\sigma_{31}^{0}(t)=\sigma_{31}^{0} f(t)$, where $f(t)$ captures its time evolution, and $\sigma_{31}^{0}$ defines its magnitude. Then, using eqn.61:

$$
\begin{aligned}
& \gamma_{1 k_{1} \ldots k_{n}}^{(n, m)}=\sigma_{33}^{0}\left[\sum_{i=1}^{n} \delta_{3 k_{i}} \prod_{j=1, j \neq i}^{n} \delta_{k_{j} u_{\pi_{i, j}}}\right] \int_{-\infty}^{t} t^{\prime m} J_{u_{1} \ldots u_{n-1}}\left(t^{\prime}\right) f\left(t^{\prime}\right) \mathrm{d} t^{\prime} \\
& \gamma_{3 k_{1} \ldots k_{n}}^{(n, m)}=\sigma_{33}^{0}\left[\sum_{i=1}^{n} \delta_{1 k_{i}} \prod_{j=1, j \neq i}^{n} \delta_{k_{j} u_{\pi_{i, j}}}\right] \int_{-\infty}^{t} t^{\prime m} J_{u_{1} \ldots u_{n-1}}\left(t^{\prime}\right) f\left(t^{\prime}\right) \mathrm{d} t^{\prime}
\end{aligned}
$$

As before, the spatial force multipoles are given by eqn.53. 

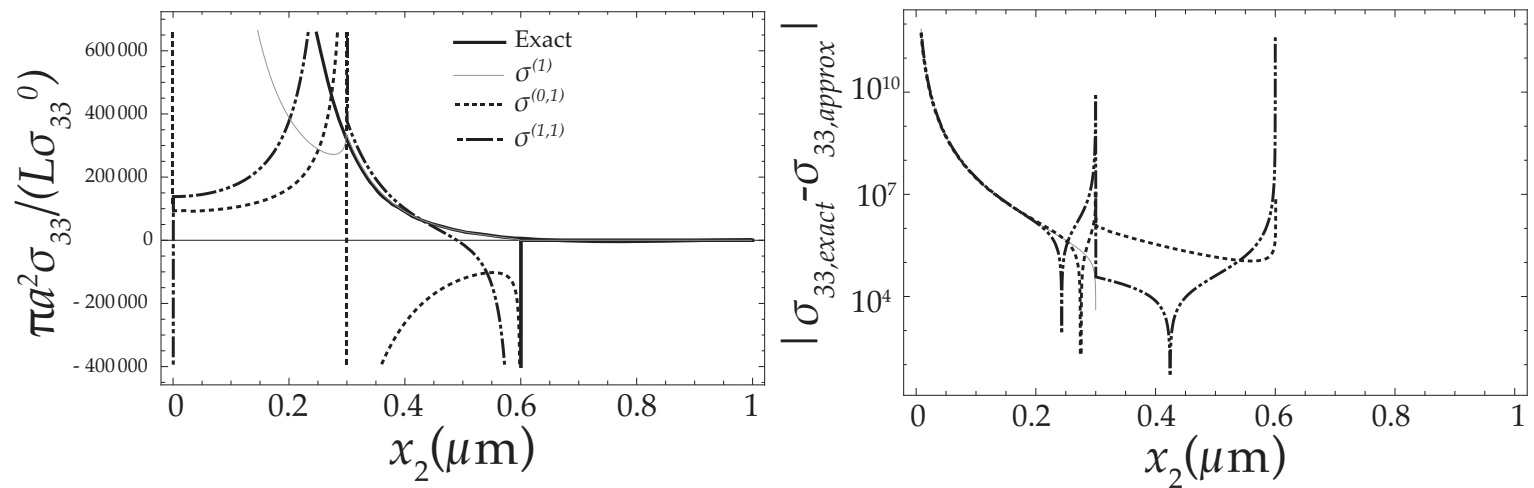

FIG. 8: Multipolar field approximations of the $\sigma_{33}$ stress field of a stationary crack that is suddenly loaded with constant pressure. The $0.1 \mathrm{~ns}$ time step is shown, for $a=1 / 6000, b=1 / 3000$.s

\section{Example}

As before, we may consider the case of a planar slit crack in mode I as an example. For simplicity and owing to space limitations, we shall consider only the case of a crack of size $L$ that is suddenly loaded with constant $\sigma_{33}^{0}$ at time $t=0^{+}$, so that $f(t)=\mathrm{H}(t)$; the analytical treatment of this problem may be found in [40]. In that case, the non-zero spatial-temporal multipolar moments are, to quadrupolar order

$$
\gamma_{23}^{(m, 1)}=\gamma_{33}^{(m, 1)}=-\sigma_{33}^{0} L \frac{t^{m+1}}{m+1}, \quad \gamma_{332}^{(m, 2)}=\gamma_{223}^{(m, 2)}=-\sigma_{33}^{0} \frac{L^{2}}{2} \frac{t^{m+1}}{m+1}, \quad \ldots
$$

To dipolar order, this leads to the following multipolar expansion of the $\sigma_{33}$ stress field, evaluated along the $x_{2}$ line (i.e., $x_{3}=0$ ):

$$
\sigma_{33}^{(0,1)}=\frac{L \sigma_{33}^{0} t\left(\sqrt{t^{2}-a^{2} x_{2}^{2}}\left(b^{2} x_{2}^{2}-2 t^{2}\right) \mathrm{H}\left(t-b \sqrt{x_{2}^{2}}\right)+\left(2 t^{2}-a^{2} x_{2}^{2}\right) \sqrt{t^{2}-b^{2} x_{2}^{2}} \mathrm{H}\left(t-a \sqrt{x_{2}^{2}}\right)\right)}{\pi a^{2} x_{2}^{4} \sqrt{t^{2}-a^{2} x_{2}^{2}} \sqrt{t^{2}-b^{2} x_{2}^{2}}}
$$

$\sigma_{33}^{(0,1)}=\frac{L \sigma_{33}^{0} t^{3}\left(\left(2 t^{2}-3 a^{2} x_{2}^{2}\right)\left(t^{2}-b^{2} x_{2}^{2}\right)^{3 / 2} \mathrm{H}\left(t-a \sqrt{x_{2}^{2}}\right)-\left(t^{2}-a^{2} x_{2}^{2}\right)^{3 / 2}\left(2 t^{2}-3 b^{2} x_{2}^{2}\right) \mathrm{H}\left(t-b \sqrt{x_{2}^{2}}\right)\right)}{2 \pi a^{2} x_{2}^{4}\left(t^{2}-a^{2} x_{2}^{2}\right)^{3 / 2}\left(t^{2}-b^{2} x_{2}^{2}\right)^{3 / 2}}$

In turn, the expansion employing the spatial multipolar moments $\gamma_{23}^{(1)}(t)=\gamma_{32}^{(1)}(t)=-\sigma_{33}^{0} L \mathrm{H}(t)$ alone leads to

$$
\sigma_{33}^{(1)}=\frac{L \sigma_{33}^{0} t\left(\sqrt{t^{2}-a^{2} x_{2}^{2}} \mathrm{H}\left(t-a x_{2}\right)-\sqrt{t^{2}-b^{2} x_{2}^{2}} \mathrm{H}\left(t-b x_{2}\right)\right)}{\pi a^{2} x_{2}^{4}}
$$

Fig.8 offers a comparison between each approximation, and with the exact solution we attained numerically employing a Newmark integration of the Navier-Lamé equations in FENicS, for a flat crack of size $L=0.1$ in a box of size $1 \times 1$, with $a=1 / 6000, b=1 / 3000, \rho=1$, with a regular mesh of $1000 \times 1000$ triangular elements. As can be seen, the spatial dipolar approximation remains accurate for the longitudinal component alone; the shear wave component, as was the case with dislocations, is incorrectly captured, the dipolar approximation predicting an inflection that is not observed in the numerical solution. The spatial-temporal dipolar approximations, in turn equally fail in capturing the singularity at the shear wave front (that is, at $x_{2} \approx 0.3 \mu \mathrm{m}$ ), and misrepresent one at the longitudinal wave front (that is, at $x_{2} \approx 0.6 \mu \mathrm{m}$ ); however, we note that increasing the temporal order of this expansion appears to improve the quality of the approximation: the first order expansion, for instance, adequately mirrors the solution's behaviour on the right hand side of the shear wave front inflection. Thus, we conclude that the dipolar approximation of the stationary crack serves to capture the longitudinal wave behaviour best. The use of the spatial-temporal multipolar moments seems again circumscribed to specific leading term analyses. 


\section{DISCUSSION AND CONCLUSIONS}

This article has provided a general theoretical framework with which to develop multipolar field expansions of dislocations and cracks. Multipolar field expansions arise from the Taylor series expansion of the Green's function; so long as the defect is represented as a force distribution, the convolution of the expanded Green's function with these force distributions results in a series representation of the defect's elastic field, where the spatial terms decrease with $1 / r^{n+1}$ for $n$ the order of the series expansion, so even at low order the long range field of the defect is captured accurately. Each successive term in the multipolar field expansion corresponds to the representation of a force dipole $(n=1)$, force quadrupole $(n=2)$, force octopole $(n=3)$, etc, that is applied in the origin, and the magnitude of which is representative of the geometry of the defect. The formulation we have derived relies on describing the dislocations and cracks as force distributions, rather than eigenstrains or eigenstresses; this is achieved employing the Burridge-Knopoff force representation theorem [22]. The force representation of defects, and its ensuing multipolar field expansion, is equivalent to the one that may achieved via eigenstresses or moment tensor representations, but it avoids further complications arising from considering higher order multipolar expansions employing moment tensor representations [6], and it retains the multipolar expansion's original meaning as a series of force multipoles applied at the origin.

We have constructed a general force representation for arbitrary three dimensional loops and cracks in an anisotropic, and applied it to obtain the general expression of their corresponding multipolar field expansions. Crucially, we have shown the $n^{\text {th }}$ order multipolar moment to be directly proportional to the $(n-1)^{\text {th }}$ moments of area enclosed by the loop, and to the material's elastic constants, leading to a very simple formulation of the quasistatic multipolar expansion. We have then studied a number of relevant cases that highlight the advantages and limitations of multipolar field expansions. In particular, we have discussed the case of planar dislocation dipoles, where we have seen the dipolar field on its own accurately describes the dipole's behaviour above distances of the same magnitude as the dipole's separation. The same sort of behaviour has been observed for the case of a three dimensional circular dislocation loop, which we have expanded to octopolar order to show that increasing the order of the expansion invariably leads to more accurate descriptions of the near field.

In studying cracks, we have focused on mode I and mode III cracks, mode II cracks being a variant of mode I cracks. We have derived the general expression of a multipolar field expansion in anisotropic, three dimensional modes I and III cracks, which has been found to be analogous to that of dislocations. Unlike dislocations however, cracks offer a far more varied casuistic, of which we have selected a number of tractable cases, studying the mode I, planar crack multipolar expansion, and that of a mode III three dimensional rectangular crack subjected to constant loading. These two cases have highlighted the high accuracy of the dipolar approximation as a measure of the crack's long range fields, and that increasing the order of the expansion results in better near field approximations. Clearly however, the multipolar expansion of a crack's elastic field does not capture the crack tip field correctly, and such expansion becomes inadequate in studying crack propagation; rather, its usefulness lies in capturing crack's long range field in applications where, either because the crack is small, or because the latter interacts with many other defects (e.g., the effect of cracks on background dislocations), this might be of interest.

Finally, we have discussed the way multipolar field expansions of dislocations and cracks may be extended to the elastodynamic continuum. We have seen that in dynamics, multipolar moments warrant at least two separate definitions. On the one hand, we have derived an expression for the space-time multipolar moments, which capture the leading terms of the time evolution of the far field. However, due to the mathematical form of the elastodynamic Green's function, we have seen that this expansion is inaccurate away from the wave fronts, and should be employed only in studying particular spectral excitations. On the other hand, we have derived a spatial multipolar moment expansion, which we have shown to display much greater accuracy in describing the far field of dislocations and cracks, even if the defects are moving. As a downside, these spatial multipolar expansions can only be computed via the time convolution of the multipolar moment with the Green's function, which becomes increasingly challenging at greater multipolar orders. We have further discussed the problematic surrounding moving defects, which limits the accuracy of the expansion to the longitudinal wave components of the fields of dislocations when the latter's motion is limited by the shear wave speed, or to low speed dislocations.

Similar results have been observed in cracks. In both cases, we have noted how the long range, longitudinal field, remains largely unaffected by the current kinematic state of the defect, because the latter propagates at a much faster speed than the defect itself. Although there exists a distinct possibility for transonic $[41,42]$ or supersonic crack propagation [40,43, 44], dislocation speeds appear to be experimentally limited to gliding at speeds below the transverse speed of sound [26, 45, 46]. This implies that the dynamic multipolar field expansions presented in this article, of much lower computational complexity, suffice to capture the longitudinal field of dislocations; given that the fields of dislocations display a strong past history effects[36], this would reduce the computational cost of evaluating their fields and 
contribute to the simplification of dynamic discrete dislocation simulations. Future work will therefore focus on implementing the multipolar expansions into elastodynamic simulations of discrete dislocation dynamics, and in their use in theoretical models of high strain rate plasticity, such as the Meyers model of shock front $[47,48]$. In the case of cracks, the multipolar expansions we have presented both in the static and the dynamic cases show a strong accuracy in quantifying their long range effects at, particularly in the elastodynamic case, much reduced computational cost. Whilst this article was aimed at providing the general formulation and justification for such analysis, cracks display a wide number of geometrical and loading features that merits further work, in particular, focusing on more comprehensive analyses of the accuracy of the approximation in specific crack geometries, and in extending them to non planar (for instance, bifurcated) cracks and to the study of cracks along bimaterial interfaces.

Multipolar field expansions of dislocations are eminently useful in a number of applications. On the one hand, similar expansions to dipolar order have already been proposed[14, 15] to accelerate discrete dislocation dynamics simulations via the fast multipole method[18]. The formulation presented here, not relying on the elastic interaction energy between dislocation loops, simplifies and generalises such efforts to higher order, to anisotropic formulations, and extends them to the elastodynamic continuum as well. Furthermore, since it does not focus on dislocation segment line integration, but on the slip surface integrals, it offers an accessible tool for theoretical studies of the long range behaviour of closed loops in three dimensions. On the other hand, there are numerous occasions where the long range field of dislocations, rather than core effects, are of interest. Such is the case of the study of interactions between dislocations and other defects such as cracks or point defects, particularly if the size of the dislocation loop is small compared to the problem's lengthscale, as would be the case for nano-loops arising from radiation damage. For instance, Dudarev and Sutton [17] offered a dipolar field approximation of the field of a nano-dislocation loops arising in radiation damage as a means studying its long range interactions with vacancy clusters.

The long range fields of cracks provided by the multipolar field expansion, albeit arguably less studied than those of dislocations in previous works, are not without interest, particularly when the size of cracks is small or the latter are acting in a heavily strained crystal. This would be the case for the study of flaw behaviour in ceramic materials[49]; the modelling of microcracks in rocks [50], semiconductors[51], or in metals undergoing frictional sliding [52]; or in the study of fragmentation, when many small scale cracks develop prior to the ultimate failure of the material[53]. Equally, the interaction between cracks and other remote defects such as inclusions [54] might be advantageously modelled by the crack's multipolar expansion. In all these cases, the internal evolution of the crack is concurrent with their interaction with multiple other cracks, often at enough distance from one another that a multipolar expansion of their mutual interactions would suffice to capture them.

Furthermore, the formulation we have presented here may be applied to the study of other extended defects, such as generalised faults[22] or voids[23], without the need to compute the elastic interaction energies, so long as the defect may be described as a plastic inclusion. As we have shown here with cracks, once their eigenstress is defined, the force representation is immediately achievable, and the multipolar field expansion would follow from the derivations we have presented in this work.

Finally, albeit centred in the elastic continuum, multipolar field expansions can be linked to atomistic efforts at modelling the core of defects. In the case of point defects, the multipolar expansion is the first step towards modelling the defect in the continuum from first principles: the forces involved in the dipoles, quadrupoles, etc of the expansion might be computed from first principles [55, 56], and then employed in the continuum multipolar expansion models [24, 57]. Having provided a comprehensive method of how to derive the multipolar field expansion of a dislocation in this work, we have effectively enabled the extension these modelling efforts to dislocations, by computing their Kanzaki forces from atomistic methods (see for instance $[58,59]$ ), and then building the corresponding multipolar model.

Acknowledgements and contributions The author conceived and wrote the whole article. The munificent support of the Master and Fellows of Trinity College Cambridge under the author's Research Fellowship is gratefully acknowledged.

[1] J. D. Jackson. Classical Electrodynamics. Wiley, New York, 1999.

[2] P. Ojeda-May and M. E. Garcia. Electric field-driven disruption of a native $\beta$-sheet protein conformation and generation of a helix-structure. Biophysical journal, 99(2):595-599, 2010.

[3] Maxim A Yurkin and Alfons G Hoekstra. The discrete dipole approximation: an overview and recent developments. Journal of Quantitative Spectroscopy and Radiative Transfer, 106(1-3):558-589, 2007.

[4] C. Teodosiu. Elastic Models of Crystal Defects. Springer-Verlag, London, 1982.

[5] R. W. Balluffi. Introduction To Elasticity Theory For Crystal Defects. World Scientific Publishing Co., Singapore, 2nd edition, 2017. 
[6] G. Backus and M. Mulcahy. Moment tensors and other phenomenological descriptions of seismic sources-i. continuous displacements. Geophysical Journal International, 46(2):341-361, 1976.

[7] G. Backus and M. Mulcahy. Moment tensors and other phenomenological descriptions of seismic sources-ii. discontinuous displacements. Geophysical Journal International, 47(2):301-329, 1976.

[8] I. A. Kunin. The theory of elastic media with microstructure and the theory of dislocations. In E. Kröner, editor, Mechanics of Generalized Continua, pages 321-329, Berlin, 1968. IUTAM, Springer-Verlag.

[9] H. S. Chen, J .H. Gilman, and A. K. Head. Dislocation multipoles and their role in strain-hardening. Journal of Applied Physics, 35(8):2502-2514, 1964.

[10] P. B. Hirsch. Work hardening. In P. B. Hirsch, editor, The Physics of Metals, volume 2, chapter 5, pages 189-246. Cambridge Univ. Press, 1975.

[11] H. Y. Wang and R. LeSar. O(N) algorithm for dislocation dynamics. Philosophical Magazine A, 71(1):149$164,1995$.

[12] A. A. Vakulenko and A. A. Kosheleva. Some problems of the theory of elasticity of composite media. Vestnik Leningradskogo Gosudarstvennogo Universiteta (Trans. Leningrad State University, in Russian), Ser. Math. Mech. Astron, (1):125-135, 1980.

[13] A. A. Kosheleva. Method of multipolar expansion in the mechanics of matrix composites. Mechanics of Composite Materials, 19(3):301-307, 1983.

[14] R. LeSar and J. M. Rickman. Multipole expansion of dislocation interactions: application to discrete dislocations. Physical Review B, 65(14):144110, 2002.

[15] D. Zhao, J. Huang, and Y. Xiang. A new version fast multipole method for evaluating the stress field of dislocation ensembles. Modelling and Simulation in Materials Science and Engineering, 18(4):045006, 2010.

[16] Z. Wang, N. Ghoniem, and R. LeSar. Multipole representation of the elastic field of dislocation ensembles. Physical Review B, 69(17):174102, 2004.

[17] S. L. Dudarev and A. P. Sutton. Elastic interactions between nano-scale defects in irradiated materials. Acta Materialia, 125:425-430, 2017.

[18] L. Greengard and V. Rokhlin. A fast algorithm for particle simulations. Journal of computational physics, $73(2): 325-348,1987$.

[19] R. De Wit. The continuum theory of stationary dislocations. Solid State Physics, 10:249-292, 1960.

[20] I. Sevostianov and M. Kachanov. Elastic fields generated by inhomogeneities: Far-field asymptotics, its shape dependence and relation to the effective elastic properties. International Journal of Solids and Structures, 48(16-17):2340-2348, 2011.

[21] V. I. Kushch and I. Sevostianov. Effective elastic moduli of a particulate composite in terms of the dipole moments and property contribution tensors. International Journal of Solids and Structures, 53:1-11, 2015.

[22] R. Burridge and L. Knopoff. Body force equivalents for seismic dislocations. Bulleting of the Seismological Society of America, 54(6):1875-1888, 1964.

[23] T. Mura. Micromechics of Defects in Solids. Kluwer Academic Publishers, Amsterdam, 2nd edition, 1982.

[24] R. W. Balluffi, S. M. Allen, and W. C. Carter. Kinetics of Materials. Wiley, Hoboken, NJ, 2005.

[25] H. Kanzaki. Point defects in face-centred cubic lattice - i distortion around defects. Journal of Physics and Chemistry of Solids, 2(1):24-36, 1957.

[26] J. P. Hirth and J. Lothe. Theory of Dislocations. John Wiley \& Sons, New York, 2nd edition, 1982.

[27] W. Rudin. Principles of Mathematical Analysis. McGraw-Hill, New York, 3rd edition, 1976.

[28] R.-J. Lange. Potential theory, path integrals and the laplacian of the indicator. Journal of High Energy Physics, 2012(11):32, 2012.

[29] E. Kröner. Kontinuumstheorie der Vemetrungen und Eigenspannungen, volume 5 of Ergebnisse der Angewandten Mathematik. Springer-Verlag, Berlin, 1958.

[30] J. R. Willis. The stress field around an elliptical crack in an anisotropic elastic medium. International Journal of Engineering Science, 6(5):253-263, 1968 .

[31] N. Langdon. Explicit expressions for stress field of a circular dislocation loop. Theoretical and applied fracture mechanics, 33(3):219-231, 2000.

[32] T. L. Anderson. Fracture Mechanics. Fundamentals and Applications. Taylor \& Francis, London, 3rd edition, 2005.

[33] M. Alnaes, J. Blechta, J. Hake, A. Johansson, B. Kehlet, A. Logg, C. Richardson, J. Ring, M. E. Rognes, and G. N. Wells. The FEniCS project version 1.5. Archive of Numerical Software, 3(100):9-23, 2015.

[34] J. D. Achenbach. Wave propagation in elastic solids. North-Holland, New York, 1973.

[35] A. C. Eringen and E. S. Suhubi. Elastodynamics, volume 2. Academic Press, New York, 1975.

[36] B. Gurrutxaga-Lerma, D. S. Balint, D. Dini, D. E. Eakins, and A. P. Sutton. A dynamic discrete dislocation plasticity method for the simulation of plastic relaxation under shock loading. Proc. Roy. Soc. A, 469:20130141, 2013.

[37] B. Gurrutxaga-Lerma, D. S. Balint, D. Dini, D. E. Eakins, and A. P. Sutton. Attenuation of the dynamic yield point of shocked aluminum using elastodynamic simulations of dislocation dynamics. Phys. Rev. Lett., 114:174301, 2015.

[38] X. Markenscoff and R. J. Clifton. The nonuniformly moving edge dislocation. J. Mech. Phys. Solids, 29(2):253-262, 1981.

[39] B Gurrutxaga-Lerma, D. S. Balint, D. Dini, and A. P. Sutton. Elastodynamic image forces on dislocations. In Proc. R. Soc. A, volume 471, page 20150433. The Royal Society, 2015.

[40] L. B. Freund. Dynamic fracture mechanics. Cambridge Univ. Press, Cambridge, UK, 1998. 
[41] A. J. Rosakis, O. Samudrala, and D. Coker. Cracks faster than the shear wave speed. Science, 284(5418):1337$1340,1999$.

[42] A. Needleman and A. J. Rosakis. The effect of bond strength and loading rate on the conditions governing the attainment of intersonic crack growth along interfaces. Journal of the Mechanics and Physics of Solids, 47(12):2411-2449, 1999.

[43] D. R. Curran, D. A. Shockey, and S. Winkler. Crack propagation at supersonic velocities. International Journal of Fracture Mechanics, 6(3):271-278, 1970.

[44] C. Behn and M. Marder. The transition from subsonic to supersonic cracks. Phil. Trans. R. Soc. A, 373(2038):20140122, 2015.

[45] X. Markenscoff and L. Ni. The transient motion of a dislocation with a ramp-like core. J. Mech. Phys. Solids, 49(1603-1619), 2001.

[46] S. Huang and X. Markenscoff. Dislocations accelerating through the shear-wave speed barrier and effect of the acceleration on the mach front curvature. International Journal of Engineering Science, 49(12):1461-1469, 2011.

[47] M. A. Meyers. A mechanism for dislocation generation in shock-wave deformation. Scripta Metallurgica, 12:21-26, 1978.

[48] M. A. Meyers, F. Gregori, B. K. Kad, M. S. Schneider, D. H. Kalantar, B. A. Remington, G. Ravichandran, T. Boehly, and J. S. Wark. Laser-induced shock compression of monocrystalline copper: characterization and analysis. Acta Materialia, 51:1211-1228, 2003.

[49] H. P. Kirchner, R. M. Gruver, and W. A. Sotter. Characteristics of flaws at fracture origins and fracture stress-flaw size relations in various ceramics. Materials Science and Engineering, 22:147-156, 1976.

[50] Robert L Kranz. Microcracks in rocks: a review. Tectonophysics, 100(1-3):449-480, 1983.

[51] Marco Paggi, Mauro Corrado, and Maria Alejandra Rodriguez. A multi-physics and multi-scale numerical approach to microcracking and power-loss in photovoltaic modules. Composite Structures, 95:630-638, 2013.

[52] Y Fu and AG Evans. Some effects of microcracks on the mechanical properties of brittle solids-i. stress, strain relations. Acta metallurgica, 33(8):1515-1523, 1985.

[53] M. A. Meyers. Dynamic Behavior of Materials. John Wiley, Hoboken, NJ, 1994.

[54] C. Atkinson. The interaction between a crack and an inclusion. International Journal of Engineering Science, 10(2):127-136, 1972.

[55] C. Domain and C. S. Becquart. Ab initio calculations of defects in fe and dilute fe-cu alloys. Physical Review $B, 65(2): 024103,2001$.

[56] C. Freysoldt, B. Grabowski, T. Hickel, J. Neugebauer, G. Kresse, A. Janotti, and C. G. Van de Walle. First-principles calculations for point defects in solids. Reviews of modern physics, 86(1):253, 2014.

[57] H. R. Schober and K. W. Ingle. Calculation of relaxation volumes, dipole tensors and kanzaki forces for point defects. Journal of Physics F: Metal Physics, 10(4):575, 1980.

[58] J. A. Caro and N. Glass. A lattice-dynamics model of the interaction of a dislocation with point defects. Journal de Physique, 45(8):1337-1345, 1984.

[59] E. Kröner. Dislocations in crystals and in continua: a confrontation. International journal of engineering science, 33(15):2127-2135, 1995.

\section{APPENDIX}

The displacement field components of the octopolar expansion of a circular dislocation loop are the following:

$$
\begin{gathered}
u_{1}=-\frac{B R^{2} x_{3}}{16(\lambda+2 \mu)\left(x_{1}^{2}+x_{2}^{2}+x_{3}^{2}\right)^{9 / 2}}\left(3 \lambda R^{2}\left(7 x_{1}^{4}+x_{1}^{2}\left(9 x_{2}^{2}-26 x_{3}^{2}\right)+2\left(x_{2}^{2}+x_{3}^{2}\right)^{2}\right)+12 \lambda x_{1}^{2}\left(x_{1}^{2}+x_{2}^{2}+x_{3}^{2}\right)^{2}+\right. \\
\left.15 \mu R^{2}\left(2 x_{1}^{4}+x_{1}^{2}\left(3 x_{2}^{2}-5 x_{3}^{2}\right)+x_{2}^{2}\left(x_{2}^{2}+x_{3}^{2}\right)\right)+4 \mu\left(x_{1}^{2}+x_{2}^{2}+x_{3}^{2}\right)^{2}\left(4 x_{1}^{2}+x_{2}^{2}+x_{3}^{2}\right)\right) \quad(82) \\
u_{2}=-\frac{3 B R^{2} x_{1} x_{2} x_{3}(\lambda+\mu)\left(5 R^{2}\left(x_{1}^{2}+x_{2}^{2}-6 x_{3}^{2}\right)+4\left(x_{1}^{2}+x_{2}^{2}+x_{3}^{2}\right)^{2}\right)}{16(\lambda+2 \mu)\left(x_{1}^{2}+x_{2}^{2}+x_{3}^{2}\right)^{9 / 2}} \\
u_{3}=-\frac{B R^{2} x_{1}}{16(\lambda+2 \mu)\left(x_{1}^{2}+x_{2}^{2}+x_{3}^{2}\right)^{9 / 2}}\left(3 \lambda x_{3}^{2}\left(5 R^{2}\left(3 x_{1}^{2}+3 x_{2}^{2}-4 x_{3}^{2}\right)+4\left(x_{1}^{2}+x_{2}^{2}+x_{3}^{2}\right)^{2}\right)+\right. \\
\left.\mu\left(3 R^{2}\left(x_{1}^{4}+2 x_{1}^{2}\left(x_{2}^{2}+6 x_{3}^{2}\right)+x_{2}^{4}+12 x_{2}^{2} x_{3}^{2}-24 x_{3}^{4}\right)+4\left(x_{1}^{2}+x_{2}^{2}+x_{3}^{2}\right)^{2}\left(x_{1}^{2}+x_{2}^{2}+4 x_{3}^{2}\right)\right)\right)
\end{gathered}
$$


The exact, full-range field of a slit crack of length $a$ is given by

$$
\begin{aligned}
\sigma_{33}= & \frac{a \sigma_{33}^{0}}{4 \pi(\nu-1) r^{4}\left(a^{2}-2 a x_{2}+r\right)^{2}}\left(a^{3}\left((2 \nu-1) x_{2}^{3}+(2 \nu-3) x_{2} x_{3}^{2}\right)\right. \\
& -a^{2}\left((6 \nu-3) x_{2}^{4}+2(2 \nu-5) x_{2}^{2} x_{3}^{2}+(1-2 \nu) x_{3}^{4}\right)+ \\
& \left.+a\left((6 \nu-3) x_{2}^{5}+2(2 \nu-7) x_{2}^{3} x_{3}^{2}-(2 \nu+3) x_{2} x_{3}^{4}\right)-r\left((2 \nu-1) x_{2}^{4}+(3-2 \nu) x_{3}^{4}-6 x_{2}^{2} x_{3}^{2}\right)\right)
\end{aligned}
$$

for $r=\sqrt{x_{2}^{2}+x_{3}^{2}}$. 